(0) Rice

a SpringerOpen Journal

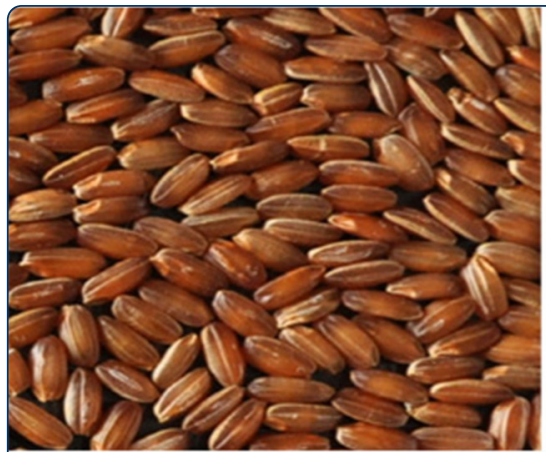

MANAVARI

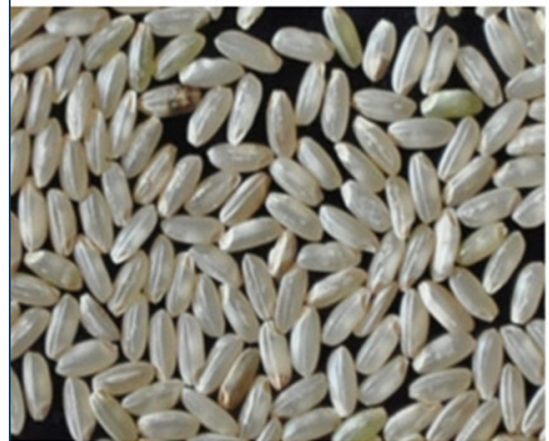

KARTHI SAMBA

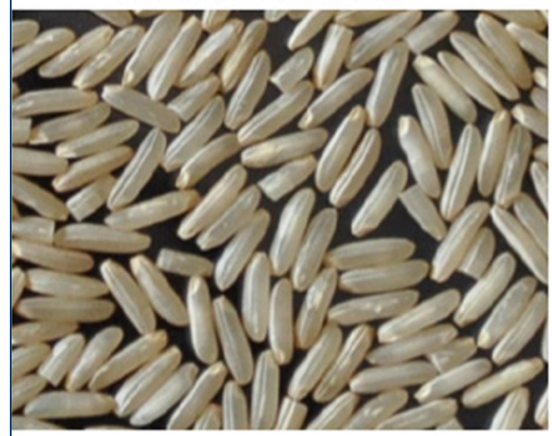

EC 729050-120988

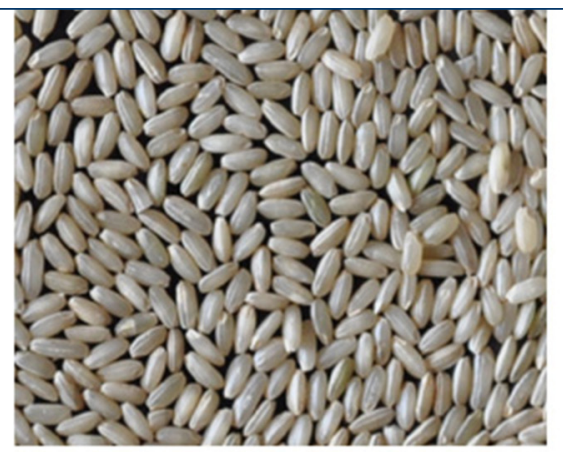

CHIR 8

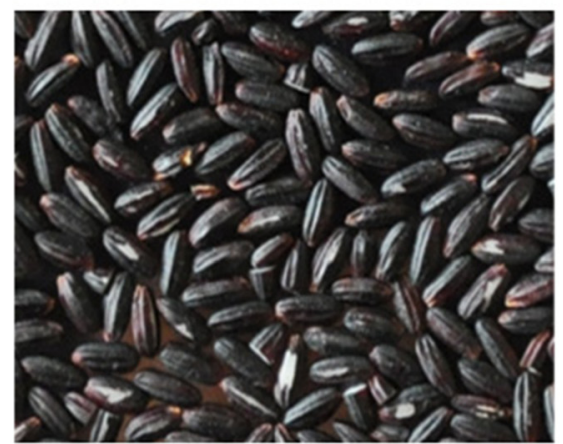

PURPLE PUTTU

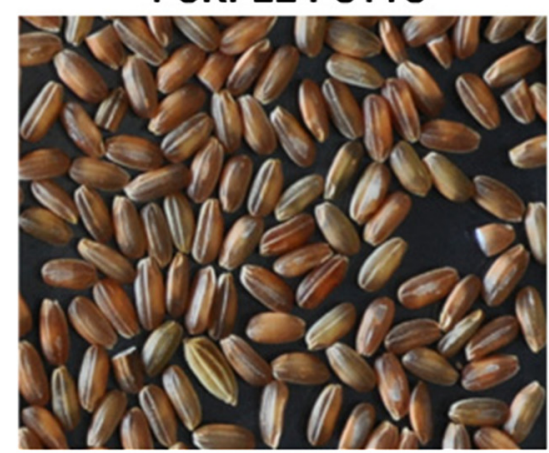

KAKARATHAN

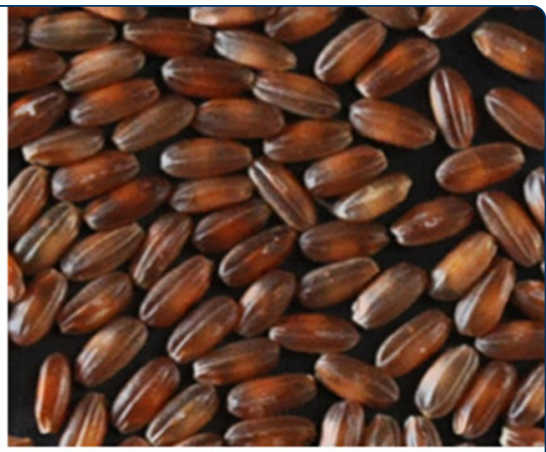

RPHP 138

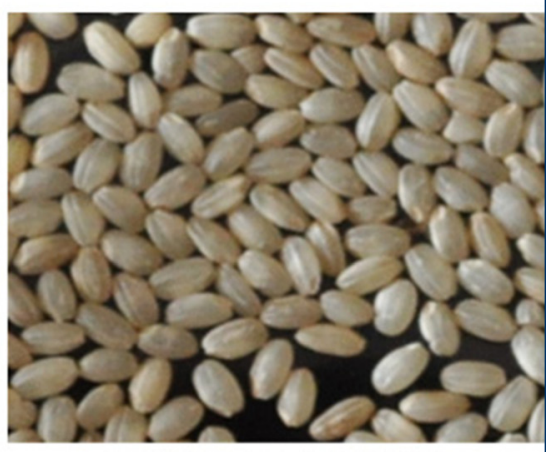

EC 728772- 117742

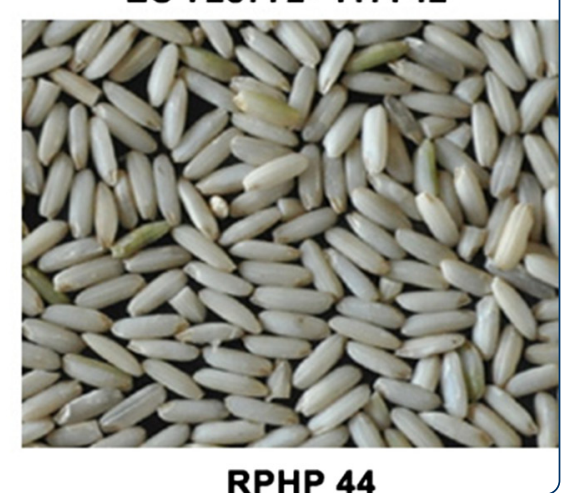

Analysis of Population Structure and Genetic Diversity in Rice Germplasm Using SSR Markers: An Initiative Towards Association Mapping of Agronomic Traits in Oryza Sativa

Nachimuthu et al.

照 Springer 


\title{
Analysis of Population Structure and Genetic Diversity in Rice Germplasm Using SSR Markers: An Initiative Towards Association Mapping of Agronomic Traits in Oryza Sativa
}

Vishnu Varthini Nachimuthu' ${ }^{1 *}$, Raveendran Muthurajan ${ }^{3}$, Sudhakar Duraialaguraja ${ }^{3}$, Rajeswari Sivakami², Balaji Aravindhan Pandian², Govinthraj Ponniah ${ }^{5}$, Karthika Gunasekaran ${ }^{4}$, Manonmani Swaminathan², Suji K K ${ }^{3}$ and Robin Sabariappan ${ }^{6}$

\begin{abstract}
Background: Genetic diversity is the main source of variability in any crop improvement program. It serves as a reservoir for identifying superior alleles controlling key agronomic and quality traits through allele mining/ association mapping. Association mapping based on LD (Linkage dis-equilibrium), non-random associations between causative loci and phenotype in natural population is highly useful in dissecting out genetic basis of complex traits. For any successful association mapping program, understanding the population structure and assessing the kinship relatedness is essential before making correlation between superior alleles and traits. The present study was aimed at evaluating the genetic variation and population structure in a collection of 192 rice germplasm lines including local landraces, improved varieties and exotic lines from diverse origin.

Results: A set of 192 diverse rice germplasm lines were genotyped using 61 genome wide SSR markers to assess the molecular genetic diversity and genetic relatedness. Genotyping of 192 rice lines using 61 SSRs produced a total of 205 alleles with the PIC value of 0.756. Population structure analysis using model based and distance based approaches revealed that the germplasm lines were grouped into two distinct subgroups. AMOVA analysis has explained that $14 \%$ of variation was due to difference between with the remaining $86 \%$ variation may be attributed by difference within groups.
\end{abstract}

Conclusions: Based on these above analysis viz., population structure and genetic relatedness, a core collection of 150 rice germplasm lines were assembled as an association mapping panel for establishing marker trait associations.

Keywords: Rice; Genetic diversity; Population structure; Polymorphism information content; Molecular variance; Association mapping

\section{Background}

Rice, being the staple food crop for more than $50 \%$ of the world population is cultivated in 163 million hectares with the production of 491 million tonnes. About $90 \%$ of the world's rice is produced in Asia and India contributes $20 \%$ of the world's production. This record level production and productivity is due to the availability

\footnotetext{
* Correspondence: popvarun@gmail.com

${ }^{1}$ Plant Molecular Biology, Plant Breeding and Genetics Divison, International Rice Research Institute, Manila, Philippines

Full list of author information is available at the end of the article
}

and exploitation of rich genetic diversity existing in rice germplasm of India. For precise genetic manipulation of complex quantitative traits like, yield, tolerance against biotic/abiotic stresses, quality etc., understanding the genetic/molecular basis of target traits needs to be investigated thoroughly.

The genetic basis of important agronomic traits has been unraveled through Quantitative Trait Loci (QTL) mapping either through linkage mapping (bi-parental mapping populations) or through LD mapping (natural populations). Although traditional linkage based QTL- 
mapping has become an important tool in gene tagging of crops, it has few limitations viz., 1) classical linkage mapping involves very high cost; 2) it has low resolution as it can resolve only a few alleles and 3) it has limitations towards fine mapping of QTLs as it needs BC-NILs. These limitations can be overcome by the LD based approach of "Association Mapping" using the natural populations. Association mapping serves as a tool to mine the elite genes by structuring the natural variation present in a germplasm. It was successfully exploited in various crops such as rice, maize, barley, durum wheat, spring wheat, sorghum, sugarcane, sugarbeet, soybean, grape, forest tree species and forage grasses (Abdurakhmonov and Abdukarimov 2008).

Before performing an association analysis in a population, it is essential to determine the population structure which can reduce type I and II errors in association mapping due to unequal allele frequency distribution between subgroups that causes spurious association between molecular markers and trait of interest (Pritchard et al. 2000). Similar attempts were recently undertaken to define population structure in rice using different germplasm lines and by developing core collection from national collections and international collections (Ebana et al. 2008; Jin et al. 2010; Zhang et al. 2011; Agrama et al. 2010 and Liakat Ali et al. 2011). Simple Sequence repeat (SSR) markers have been commonly used in genetic diversity studies in rice because of high level of polymorphism which helps to establish the relationship among the individuals even with less number of markers (McCouch et al. 1997). For similar studies, SSR markers were used alone by Jin et al. (2010); Hesham et al. (2008); Sow et al. (2014); Das et al. (2013) and Choudhury et al. (2013) or along with SNP markers by Courtois et al. (2012) and Zhao et al. (2011). The objectives of this present study were to evaluate the genetic variation and to examine the population structure of 192 rice germplasm accessions that comprises of local landraces, improved varieties and exotic lines from diverse origin.

\section{Results}

\section{Genetic Diversity}

All the 192 rice germplasm lines were genotyped using 61 SSR (microsatellite) markers which produced a total of 205 alleles (Additional file 1: Figure S1). Among these 205 alleles, $5 \%$ were considered as rare (showed an allele frequency of $<5 \%$ ). The number of alleles per loci varied from 2 to 7 with an average of 3 alleles per locus. The highest number of alleles were detected for the loci RM316 (7) and the lowest was detected for a group of markers viz., RM171, RM284, RM455, RM514, RM277, RM 5795, HvSSR0247, RM 559, RM416 and RM1227. PIC value represents the relative informativeness of each marker and in the present study, the average PIC value was found to be 0.468 . The highest genetic diversity is explained by the landraces included in this study with the mean PIC value of 0.416 . PIC values ranged between 0.146 for RM17616 to 0.756 for RM316. Heterozygosity was found to be very low which may be due to autogamous nature of rice. Expected heterozygosity or Gene diversity $\left(\mathrm{H}_{\mathrm{e}}\right)$ computed according to Nei (1973) varied from 0.16 (RM17616) to 0.75 (RM287) with the average of 0.52 (Table 1).

\section{STRUCTURE Analysis}

Population structure of the 192 germplasm lines was analysed by Bayesian based approach. The estimated membership fractions of 192 accessions for different values of $\mathrm{k}$ ranged between 2 and 5 (Fig. 1). The log likelihood revealed by structure showed the optimum value as $2(K=2)$. Similarly the maximum of adhoc measure $\Delta \mathrm{K}$ was found to be $K=2$ (Fig. 2), which indicated that the entire population can be grouped into two subgroups (SG1 and SG2). Based on the membership fractions, the accessions with the probability of $\geq 80 \%$ were assigned to corresponding subgroups with others categorized as admixture (Fig. 3).

SG1 consisted of 134 accessions with most of the landraces and varieties of Indian origin and SG2 consisted of 38 accessions which composed of non Indian accessions. Twenty accessions were retained to be admixture. The subgroup SG1 was dominated by indica subtype whereas the subgroup SG2 consisted mostly of japonica group. When the number of subgroups increased from two to five, the accessions in both the subgroups were classified into sub-sub groups (Table 2). As SG1 consisted of 134 accessions mostly of Indian origin, an independent STRUCTURE analysis was performed for this subgroup. $\Delta \mathrm{K}$ showed its maximum value for $\mathrm{K}=3$ which indicated that SG1 could be further classified into three sub-sub groups (Fig. 4). The differentiation in origin and seasonal differentiation of rice varieties contributed for this clustering.

Clustering analysis based on Unweighted Pair Group Method with Arithmetic Mean (UPGMA) method using DARwin separated the accessions into two main groups which showed similar results as STRUCTURE analysis. The group I in UPGMA tree consists of both indigenous and agronomically improved varieties whereas the other group consists of exotic accessions. In UPGMA tree, the accessions within group 1 and 2 clustered into smaller sub groups based on their origin and types. Most of the landraces and varieties have been clustered in upper branches of the tree whereas the exotic accessions have been clustered in lower branches of the tree (Fig 5). Hence the clustering analysis by two classification methods revealed high level of similarity in clustering the genotypes. PCoA was used to characterize the 
Table 1 Details of SSR loci used for genotyping in the 192 rice accessions and their genetic diversity parameters

\begin{tabular}{|c|c|c|c|c|c|c|c|c|c|}
\hline S. no & Marker & $\begin{array}{l}\text { Chromosome } \\
\text { no. }\end{array}$ & SSR MOTIF & $\begin{array}{l}\text { Min molecular } \\
\text { weight }\end{array}$ & $\begin{array}{l}\text { Maximum } \\
\text { molecular weight }\end{array}$ & $\begin{array}{l}\text { Number } \\
\text { of alleles }\end{array}$ & Gene diversity & Heterozygosity & PIC value \\
\hline 1 & RM237 & 1 & (CT)18 & 110 & 143 & 4 & 0.61 & 0.89 & 0.545 \\
\hline 2 & $\mathrm{RM} 1$ & 1 & $(G A) 26$ & 70 & 105 & 3 & 0.63 & 0.12 & 0.552 \\
\hline 3 & RM5 & 1 & $(G A) 14$ & 105 & 115 & 3 & 0.64 & 0.6 & 0.557 \\
\hline 4 & RM312 & 1 & (ATTT)4(GT)9 & 95 & 105 & 3 & 0.3 & 0.03 & 0.281 \\
\hline 5 & RM283 & 1 & $(G A) 18$ & 149 & 155 & 3 & 0.42 & 0.02 & 0.377 \\
\hline 6 & RM452 & 2 & (GTC)9 & 195 & 245 & 3 & 0.54 & 0.83 & 0.448 \\
\hline 7 & HvSSR0247 & 2 & & 395 & 400 & 2 & 0.5 & 0.18 & 0.373 \\
\hline 8 & RM555 & 2 & $(A G) 11$ & 135 & 145 & 3 & 0.59 & 0.04 & 0.517 \\
\hline 9 & RM211 & 2 & (TC)3A(TC)18 & 140 & 160 & 3 & 0.52 & 0.08 & 0.463 \\
\hline 10 & RM324 & 2 & (CAT) 21 & 135 & 180 & 5 & 0.74 & 0.06 & 0.695 \\
\hline 11 & RM514 & 3 & $(\mathrm{AC}) 12$ & 245 & 252 & 2 & 0.19 & 0 & 0.171 \\
\hline 12 & RM55 & 3 & $(G A) 17$ & 220 & 225 & 3 & 0.44 & 0.07 & 0.4 \\
\hline 13 & RM231 & 3 & (CT) 16 & 170 & 200 & 3 & 0.59 & 0.12 & 0.511 \\
\hline 14 & RM416 & 3 & $(G A) 9$ & 110 & 115 & 2 & 0.42 & 0.01 & 0.335 \\
\hline 15 & RM442 & 3 & $(\mathrm{AAG}) 10$ & 260 & 275 & 3 & 0.5 & 0.03 & 0.448 \\
\hline 16 & RM 16643 & 4 & $(G G G A) 5$ & 165 & 200 & 5 & 0.73 & 0.05 & 0.685 \\
\hline 17 & RM 559 & 4 & $(A A C A) 6$ & 160 & 165 & 2 & 0.39 & 0.01 & 0.311 \\
\hline 18 & RM17377 & 4 & $(A G) 25$ & 140 & 175 & 4 & 0.67 & 0.04 & 0.625 \\
\hline 19 & RM7585 & 4 & (TCTT)6 & 140 & 160 & 4 & 0.46 & 0.02 & 0.422 \\
\hline 20 & RM17616 & 4 & (TC)14 & 165 & 180 & 3 & 0.16 & 0 & 0.146 \\
\hline 21 & RM413 & 5 & $(A G) 11$ & 75 & 100 & 4 & 0.59 & 0.25 & 0.548 \\
\hline 22 & RM178 & 5 & $(\mathrm{GA}) 5(\mathrm{AG}) 8$ & 110 & 115 & 3 & 0.39 & 0.04 & 0.35 \\
\hline 23 & RM 161 & 5 & $(A G) 20$ & 160 & 180 & 3 & 0.29 & 0.04 & 0.258 \\
\hline 24 & RM7293 & 5 & (ATGT)6 & 140 & 150 & 3 & 0.64 & 0.1 & 0.558 \\
\hline 25 & RM1024 & 5 & $(\mathrm{AC}) 13$ & 125 & 140 & 3 & 0.32 & 0.02 & 0.298 \\
\hline 26 & RM 162 & 6 & $(A C) 20$ & 220 & 240 & 3 & 0.37 & 0.03 & 0.34 \\
\hline 27 & RM7434 & 6 & (GTAT)10 & 120 & 145 & 5 & 0.66 & 0.19 & 0.614 \\
\hline 28 & RM19620 & 6 & (GTG)7 & 160 & 177 & 3 & 0.21 & 0.03 & 0.204 \\
\hline 29 & RM5963 & 6 & $(C A G) 9$ & 160 & 175 & 3 & 0.48 & 0.15 & 0.38 \\
\hline 30 & RM11 & 7 & $(G A) 17$ & 120 & 150 & 4 & 0.71 & 0.72 & 0.661 \\
\hline 31 & RM118 & 7 & $(\mathrm{GA}) 8$ & 155 & 185 & 4 & 0.62 & 0.77 & 0.543 \\
\hline 32 & $\mathrm{RM} 125$ & 7 & $(\mathrm{GCT}) 8$ & 105 & 130 & 4 & 0.61 & 0.89 & 0.544 \\
\hline 33 & RM455 & 7 & $($ TTCT)5 & 130 & 135 & 2 & 0.24 & 0.02 & 0.208 \\
\hline 34 & HvSSR0740 & 7 & & 340 & 400 & 4 & 0.7 & 0.21 & 0.65 \\
\hline 35 & RM44 & 8 & $(G A) 16$ & 95 & 107 & 4 & 0.62 & 0.77 & 0.559 \\
\hline 36 & RM433 & 8 & $(A G) 13$ & 235 & 270 & 3 & 0.55 & 0.81 & 0.446 \\
\hline 37 & RM447 & 8 & (CTT)8 & 105 & 120 & 4 & 0.64 & 0.16 & 0.572 \\
\hline 38 & RM284 & 8 & $(\mathrm{GA}) 8$ & 140 & 145 & 2 & 0.21 & 0.02 & 0.189 \\
\hline 39 & RM408 & 8 & (CT) 13 & 120 & 125 & 3 & 0.52 & 0.01 & 0.465 \\
\hline 40 & RM25 & 8 & $(G A) 18$ & 120 & 140 & 4 & 0.73 & 0.37 & 0.679 \\
\hline 41 & RM256 & 8 & $(\mathrm{CT}) 21$ & 125 & 140 & 4 & 0.73 & 0 & 0.681 \\
\hline 42 & RM105 & 9 & $(\mathrm{CCT}) 6$ & 100 & 140 & 3 & 0.41 & 0.48 & 0.37 \\
\hline 43 & RM107 & 9 & $(G A) 7$ & 280 & 300 & 3 & 0.48 & 0 & 0.425 \\
\hline
\end{tabular}


Table 1 Details of SSR loci used for genotyping in the 192 rice accessions and their genetic diversity parameters (Continued)

\begin{tabular}{|c|c|c|c|c|c|c|c|c|c|}
\hline 44 & RM 215 & 9 & (CT) 16 & 140 & 150 & 3 & 0.6 & 0.01 & 0.528 \\
\hline 45 & RM 316 & 9 & (GT)8-(TG)9(TTG)4(TG)4 & 160 & 235 & 7 & 0.79 & 0.75 & 0.756 \\
\hline 46 & RM205 & 9 & $(\mathrm{CT}) 25$ & 110 & 140 & 4 & 0.72 & 0 & 0.665 \\
\hline 47 & RM171 & 10 & (GATG)5 & 320 & 330 & 2 & 0.24 & 0.02 & 0.211 \\
\hline 48 & $\mathrm{RM} 271$ & 10 & $(G A) 15$ & 90 & 99 & 3 & 0.66 & 0.19 & 0.588 \\
\hline 49 & RM590 & 10 & $(\mathrm{TCT}) 10$ & 120 & 140 & 4 & 0.57 & 0.04 & 0.516 \\
\hline 50 & RM474 & 10 & (AT) 13 & 240 & 280 & 3 & 0.61 & 0 & 0.537 \\
\hline 51 & RM222 & 10 & (CT)18 & 200 & 220 & 3 & 0.63 & 0.02 & 0.557 \\
\hline 52 & RM144 & 11 & (ATT) 11 & 160 & 240 & 5 & 0.69 & 0.18 & 0.644 \\
\hline 53 & RM287 & 11 & $(G A) 21$ & 95 & 110 & 5 & 0.75 & 0.2 & 0.706 \\
\hline 54 & RM 536 & 11 & $(\mathrm{CT}) 16$ & 240 & 270 & 5 & 0.74 & 0.06 & 0.701 \\
\hline 55 & RM224 & 11 & $(\mathrm{AAG}) 8(\mathrm{AG}) 13$ & 120 & 155 & 5 & 0.65 & 0.07 & 0.617 \\
\hline 56 & RM206 & 11 & $(\mathrm{CT}) 21$ & 130 & 145 & 4 & 0.34 & 0 & 0.319 \\
\hline 57 & RM277 & 12 & $(\mathrm{GA}) 11$ & 115 & 120 & 2 & 0.45 & 0.08 & 0.35 \\
\hline 58 & RM 5795 & 12 & $(A G C) 8$ & 140 & 145 & 2 & 0.5 & 0.03 & 0.374 \\
\hline 59 & RM1227 & 12 & $(A G) 15$ & 160 & 180 & 2 & 0.31 & 0.02 & 0.262 \\
\hline 60 & $\mathrm{RM} 20 \mathrm{~A}$ & 12 & (ATT) 14 & 220 & 240 & 3 & 0.54 & 0 & 0.476 \\
\hline \multirow[t]{2}{*}{61} & RM2197 & 12 & (AT) 23 & 135 & 140 & 2 & 0.44 & 0 & 0.341 \\
\hline & Average & & & & & 3 & 0.52 & 0.18 & 0.468 \\
\hline
\end{tabular}

subgroups of the germplasm set. A two- dimensional scatter plot involving all 192 accessions has shown that the first two PCA axes accounted for 12.6 and $4.9 \%$ of the genetic variation among populations (Fig 6).

\section{Genetic Variance Analysis}

The hierarchial distribution of molecular variance by AMOVA and pair-wise analysis revealed highly significant genetic differentiation among the groups. It revealed that $14 \%$ of the total variation was between the groups, while $86 \%$ was among individuals within groups (Tables 3 and 4). Calculation of Wright's F statistic at all SSR loci revealed that $F_{I S}$ was 0.50 and $F_{I T}$ was 0.56 . Determination of $\mathrm{F}_{\mathrm{ST}}$ for the polymorphic loci across all accessions has shown $\mathrm{F}_{\mathrm{ST}}$ as 0.14 which implies high genetic variation (Table 4). The pairwise $\mathrm{F}_{\mathrm{ST}}$ estimate among sub-groups has indicated that the two groups are significantly different from each other (Table 3).

\section{Discussion}

Genetic diversity is the key determinant of germplasm utilization in crop improvement. Population with high level of genetic variation is the valuable resource for broadening the genetic base in any breeding program. The panel of 192 accessions in this study with landraces, varieties as well as breeding lines has different salient agronomic traits. Few landraces included in this study i.e., Mappillai samba (Krishnanunni et al. 2015), Jyothi,
Njavara (Deepa et al. 2008), Kavuni (Valarmathi et al. 2015) derived breeding line has therapeutic properties. Many lines included in this study are drought tolerant (Nootripathu, Norungan, Vellaikudaivazhai, kallundaikar, kodai, kalinga 3, Kinandang patong, azucena, mattaikar, IR65907-116-1, karuthakar, mattakuruvai, manavari, kallundai, kodaikulathan, kattikar, poongar, thogai samba, vellaikattai, kattukuthalam, kalvalai, chivapu chithiraikar, vellai chithiraikar, kudaivazhai and murugankar). Few lines have significant level of micronutrients in it (Nachimuthu et al. 2014). This panel has its importance because of its major component as traditional landraces with valuable agronomic traits that are cultivated in the small pockets of Tamil Nadu, India.

Molecular markers help us to understand the level of genetic diversity that exists among traditional races, varieties and exotic accessions which can be exploited in rice breeding programs. The genetic architecture of diverse germplasm lines can be precisely estimated by assessing the STRUCTURE of the population using molecular markers viz., SSRs or SNPs etc., (Horst and Wenzel 2007; Powell et al. 1996; Varshney et al. 2007). In this study, the genetic diversity among the accessions was evaluated by model based clustering and distance based clustering approach using the SSR genotypic data.

Regarding genetic divergence of the population consisting of local landraces, exotic cultivars and breeding lines, 61 polymorphic markers have detected a total of 205 


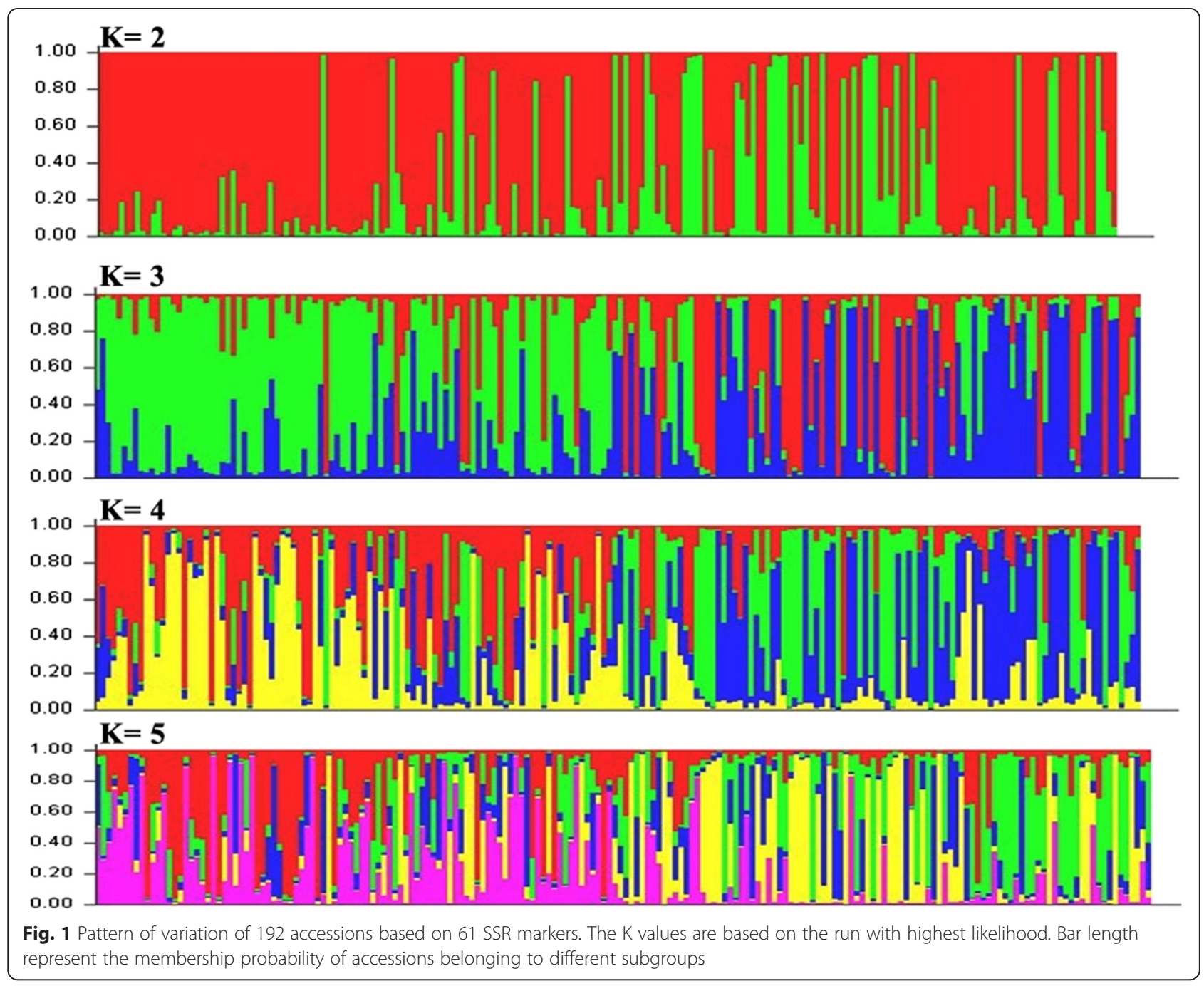

alleles across 192 individuals. The number of alleles varied from 2 to 7 per locus and the average was 3 alleles per locus. Several previous reports have indicated the number of alleles per locus, polymorphic information content and gene diversity of 4.8-14.0, 0.63-0.70 and 6.2-6.8 respectively (Garris et al. 2005; Ram et al. 2007). In the current study, the average number of alleles (3 alleles/locus) is slightly lesser than the average number of alleles (3.88 alleles/ locus) reported by Zhang et al. (2011) in rice core collection with 150 rice varieties from south Asia and Brazil and Jin et al. (2010) who has reported the average alleles per locus as 3.9 in 416 rice accessions collected from China. Using three sets of germplasm lines (Thai (47), IRRI germplasm (53) amd other Oryza species (5)), Chakhonkaen et al. (2012) has reported 127 alleles for all loci, with a mean of 6.68 alleles per locus, and a mean Polymorphic Information Content (PIC) of 0.440 by screening with 19 InDel markers.

Chen et al. (2011) has reported the average gene diversity of 0.358 and polymorphic information content of
0.285 from 300 rice accessions from different rice growing areas of the world with 372 SNP markers. The gene diversity detected in this study (0.52) is comparable to overall gene diversity of rice core collection (0.544) from China, North Korea, Japan, Philippines, Brazil, Celebes, Java, Oceanina and Vietnam (Zhang et al. 2011) and it is higher than US accession panel with average gene diversity of 0.43 (Agrama and Eizenga 2008) and Chinese rice accession panel by Jin et al. (2010) with the average gene diversity of 0.47 . The gene diversity reported in our study is lesser than gene diversity $(0.68)$ reported by (Liakat Ali et al. 2011). Most of the diversity panel with global accessions has the gene diversity of 0.5 to 0.7 (Garris et al. 2005; Liakat Ali et al. 2011; Ni et al. 2002). These results on global accessions help to infer that this diversity panel of 192 germplasm lines represents a large proportion of the genetic diversity that exists in major rice growing Asian continent.

The PIC value was 0.468 which varied from 0.146 for RM17616 with only 2 two alleles to 0.756 for RM316 

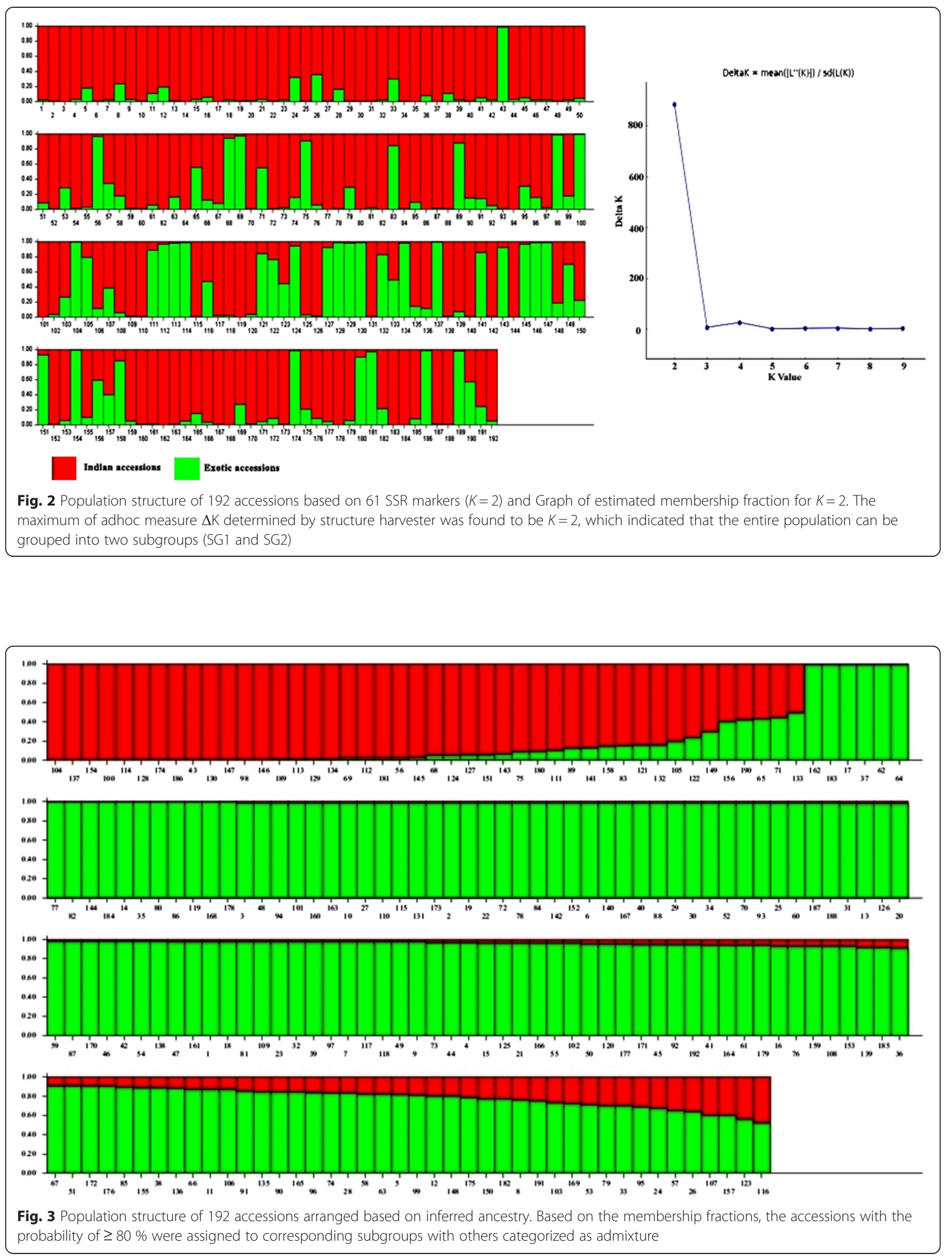
Table 2 Population structure group of accessions based on Inferred ancestry values

\begin{tabular}{|c|c|c|c|c|c|}
\hline \multirow[t]{2}{*}{ G. no. } & \multirow[t]{2}{*}{ Genotypes } & \multicolumn{2}{|c|}{ Inferred ancestry } & \multirow[t]{2}{*}{ Structure group } & \multirow[t]{2}{*}{ Subtype } \\
\hline & & $\overline{\mathrm{Q} 1}$ & Q2 & & \\
\hline$\overline{R G 1}$ & Mapillai samba & 0.977 & 0.023 & SG1 & Indica \\
\hline $\mathrm{RG} 2$ & CK 275 & 0.991 & 0.009 & SG1 & Indica \\
\hline RG3 & Senkar & 0.992 & 0.008 & SG1 & Indica \\
\hline RG4 & Murugankar & 0.964 & 0.036 & SG1 & Indica \\
\hline RG5 & CHIR 6 & 0.811 & 0.189 & SG1 & Indica \\
\hline RG6 & CHIR 5 & 0.989 & 0.011 & SG1 & Indica \\
\hline RG7 & Kudai vazhai & 0.975 & 0.025 & SG1 & Indica \\
\hline RG8 & CHIR 8 & 0.759 & 0.241 & SG1 & Indica \\
\hline RG9 & Kuruvai kalanjiyam & 0.971 & 0.029 & SG1 & Indica \\
\hline RG10 & Nava konmani & 0.99 & 0.01 & SG1 & Indica \\
\hline RG11 & CHIR 10 & 0.869 & 0.131 & SG1 & Indica \\
\hline $\mathrm{RG} 12$ & Vellai chithiraikar & 0.802 & 0.198 & SG1 & Indica \\
\hline RG13 & CHIR 2 & 0.983 & 0.017 & SG1 & Indica \\
\hline RG14 & Jothi & 0.992 & 0.008 & SG1 & indica \\
\hline RG15 & Palkachaka & 0.962 & 0.038 & SG1 & indica \\
\hline RG16 & Thooyala & 0.934 & 0.066 & SG1 & indica \\
\hline RG17 & Chivapu chithiraikar & 0.994 & 0.006 & SG1 & indica \\
\hline RG18 & CHIR 11 & 0.976 & 0.024 & SG1 & indica \\
\hline RG19 & Koolavalai & 0.99 & 0.01 & SG1 & indica \\
\hline RG20 & Kalvalai & 0.982 & 0.018 & SG1 & indica \\
\hline RG21 & Mohini samba & 0.963 & 0.037 & SG1 & indica \\
\hline RG22 & IR 36 & 0.989 & 0.011 & SG1 & indica \\
\hline $\mathrm{RG} 23$ & Koombalai & 0.975 & 0.025 & SG1 & indica \\
\hline RG24 & Tadukan & 0.674 & 0.326 & $A D$ & indica \\
\hline$R G 25$ & Sorna kuruvai & 0.986 & 0.014 & SG1 & indica \\
\hline RG26 & Rascadam & 0.637 & 0.363 & $A D$ & indica \\
\hline $\mathrm{RG} 27$ & Muzhi karuppan & 0.991 & 0.009 & SG1 & indica \\
\hline $\mathrm{RG} 28$ & Kaatukuthalam & 0.828 & 0.172 & SG1 & indica \\
\hline RG29 & Vellaikattai & 0.987 & 0.013 & SG1 & indica \\
\hline RG30 & Poongar & 0.987 & 0.013 & SG1 & indica \\
\hline RG31 & Chinthamani & 0.985 & 0.015 & SG1 & indica \\
\hline RG32 & Thogai samba & 0.975 & 0.025 & SG1 & indica \\
\hline RG33 & Malayalathan samba & 0.701 & 0.299 & $A D$ & indica \\
\hline RG34 & RPHP 125 & 0.986 & 0.014 & SG1 & indica \\
\hline RG35 & CK 143 & 0.993 & 0.007 & SG1 & indica \\
\hline RG36 & Kattikar & 0.913 & 0.087 & SG1 & indica \\
\hline RG37 & Shenmolagai & 0.994 & 0.006 & SG1 & indica \\
\hline RG38 & Velli samba & 0.887 & 0.113 & SG1 & indica \\
\hline RG39 & Kaatu ponni & 0.975 & 0.025 & SG1 & indica \\
\hline RG40 & kakarathan & 0.989 & 0.011 & SG1 & indica \\
\hline RG41 & Godavari samba & 0.941 & 0.059 & SG1 & indica \\
\hline RG42 & Earapalli samba & 0.978 & 0.022 & SG1 & indica \\
\hline RG43 & RPHP 129 & 0.01 & 0.99 & SG2 & indica \\
\hline
\end{tabular}


Table 2 Population structure group of accessions based on Inferred ancestry values (Continued)

\begin{tabular}{|c|c|c|c|c|c|}
\hline RG44 & Mangam samba & 0.968 & 0.032 & $\mathrm{SG} 1$ & indica \\
\hline RG45 & RPHP 105 & 0.943 & 0.057 & SG1 & indica \\
\hline RG46 & IG 4(EC 729639- 121695) & 0.977 & 0.023 & $\mathrm{SG} 1$ & indica \\
\hline RG47 & Machakantha & 0.976 & 0.024 & SG1 & indica \\
\hline RG48 & Kalarkar & 0.992 & 0.008 & SG1 & indica \\
\hline RG49 & Valanchennai & 0.972 & 0.028 & SG1 & indica \\
\hline RG50 & Sornavari & 0.957 & 0.043 & $\mathrm{SG1}$ & indica \\
\hline RG51 & RPHP 134 & 0.909 & 0.091 & SG1 & indica \\
\hline RG52 & ARB 58 & 0.987 & 0.013 & SG1 & indica \\
\hline RG53 & IR 68144-2B-2-2-3-1-127 & 0.708 & 0.292 & $A D$ & indica \\
\hline RG54 & PTB 19 & 0.981 & 0.019 & SG1 & indica \\
\hline RG55 & IG 67(EC 729050- 120988) & 0.957 & 0.043 & $\mathrm{SG} 1$ & indica \\
\hline RG56 & RPHP 59 & 0.031 & 0.969 & SG2 & Aromatic \\
\hline RG57 & RPHP 103 & 0.656 & 0.344 & $A D$ & Aromatic \\
\hline RG58 & Kodaikuluthan & 0.828 & 0.172 & SG1 & indica \\
\hline RG59 & RPHP 68 & 0.981 & 0.019 & $\mathrm{SG} 1$ & indica \\
\hline RG60 & Rama kuruvaikar & 0.985 & 0.015 & SG1 & indica \\
\hline RG61 & Kallundai & 0.939 & 0.061 & SG1 & indica \\
\hline RG62 & Purple puttu & 0.994 & 0.006 & SG1 & indica \\
\hline RG63 & IG 71(EC 728651- 117588) & 0.823 & 0.177 & SG1 & aus \\
\hline RG64 & Ottadaiyan & 0.994 & 0.006 & SG1 & indica \\
\hline RG65 & IG 56(EC 728700- 117658 & 0.435 & 0.565 & $A D$ & Aromatic \\
\hline RG66 & Jeevan samba & 0.876 & 0.124 & $\mathrm{SG} 1$ & indica \\
\hline RG67 & RPHP 106 & 0.915 & 0.085 & SG1 & indica \\
\hline RG68 & IG 63(EC 728711- 117674) & 0.049 & 0.951 & SG2 & Tropical Japonica \\
\hline RG69 & RPHP 48 & 0.025 & 0.975 & SG2 & Aromatic \\
\hline RG70 & Karthi samba & 0.987 & 0.013 & $\mathrm{SG} 1$ & indica \\
\hline RG71 & IG 27(IC 0590934- 121255) & 0.444 & 0.556 & $A D$ & indica \\
\hline RG72 & Aarkadu kichili & 0.99 & 0.01 & $\mathrm{SG} 1$ & indica \\
\hline RG73 & Kunthali & 0.969 & 0.031 & SG1 & indica \\
\hline RG74 & ARB 65 & 0.83 & 0.17 & SG1 & indica \\
\hline RG75 & IG 21(EC 729334- 121355) & 0.091 & 0.909 & SG2 & japonica \\
\hline RG76 & Matta kuruvai & 0.934 & 0.066 & $\mathrm{SG1}$ & indica \\
\hline RG77 & Karuthakar & 0.994 & 0.006 & SG1 & indica \\
\hline RG78 & RPHP 165 & 0.99 & 0.01 & SG1 & indica \\
\hline RG79 & Manavari & 0.704 & 0.296 & $A D$ & indica \\
\hline RG80 & IG 66(EC 729047- 120985) & 0.992 & 0.008 & SG1 & indica \\
\hline RG81 & CB-07-701-252 & 0.977 & 0.023 & $\mathrm{SG} 1$ & indica \\
\hline RG82 & Thooyamalli & 0.994 & 0.006 & SG1 & indica \\
\hline RG83 & RPHP 93 & 0.153 & 0.847 & SG2 & indica \\
\hline RG84 & Velsamba & 0.99 & 0.01 & SG1 & indica \\
\hline RG85 & RPHP 104 & 0.898 & 0.102 & $\mathrm{SG} 1$ & indica \\
\hline RG86 & RPHP 102 & 0.993 & 0.007 & SG1 & indica \\
\hline RG87 & IG 40(EC 728740- 117705) & 0.98 & 0.02 & SG1 & indica \\
\hline RG88 & Saranga & 0.988 & 0.012 & SG1 & indica \\
\hline
\end{tabular}


Table 2 Population structure group of accessions based on Inferred ancestry values (Continued)

\begin{tabular}{|c|c|c|c|c|c|}
\hline RG89 & IR 83294-66-2-2-3-2 & 0.125 & 0.875 & SG2 & japonica \\
\hline RG90 & IG 61(EC 728731- 117696) & 0.843 & 0.157 & SG1 & indica \\
\hline RG91 & IG 23(EC 729391- 121419) & 0.852 & 0.148 & SG1 & Aus \\
\hline RG92 & IG 49(EC 729102- 121052) & 0.945 & 0.055 & SG1 & indica \\
\hline RG93 & uppumolagai & 0.987 & 0.013 & SG1 & indica \\
\hline RG94 & Karthigai samba & 0.993 & 0.007 & SG1 & indica \\
\hline RG95 & Jeeraga samba & 0.685 & 0.315 & SG1 & indica \\
\hline RG96 & $\mathrm{RP}-\mathrm{BIO}-226$ & 0.833 & 0.167 & SG1 & indica \\
\hline RG97 & Varigarudan samba & 0.975 & 0.025 & SG1 & indica \\
\hline RG98 & IG 5(EC 729642- 121698) & 0.012 & 0.988 & $\mathrm{SG} 2$ & japonica \\
\hline RG99 & IG 31(EC 728844- 117829) & 0.813 & 0.187 & SG1 & indica \\
\hline RG100 & IG 7(EC 729598- 121648) & 0.008 & 0.992 & SG2 & japonica \\
\hline RG101 & RPHP 52 & 0.991 & 0.009 & SG1 & indica \\
\hline RG102 & Varakkal & 0.958 & 0.042 & SG1 & indica \\
\hline RG103 & Mattaikar & 0.732 & 0.268 & $\mathrm{AD}$ & indica \\
\hline RG104 & IG 53(EC 728752- 117719) & 0.005 & 0.995 & $\mathrm{SG} 2$ & Temperate japonica \\
\hline RG105 & IG 6(EC 729592- 121642) & 0.204 & 0.796 & SG2 & Temperate japonica \\
\hline RG106 & Katta samba & 0.872 & 0.128 & SG1 & indica \\
\hline RG107 & $\mathrm{RH} 2-\mathrm{SM}-1-2-1$ & 0.606 & 0.394 & $A D$ & indica \\
\hline RG108 & Red sirumani & 0.93 & 0.07 & SG1 & indica \\
\hline RG109 & Vadivel & 0.977 & 0.023 & SG1 & indica \\
\hline RG110 & Norungan & 0.991 & 0.009 & SG1 & indica \\
\hline RG111 & IG 20(EC 729293- 121310) & 0.113 & 0.887 & SG2 & indica \\
\hline RG112 & IG 35(EC 728858- 117843) & 0.027 & 0.973 & $\mathrm{SG} 2$ & japonica \\
\hline RG113 & IG 45(EC 728768- 117736) & 0.017 & 0.983 & SG2 & japonica \\
\hline RG114 & RPHP 159 & 0.008 & 0.992 & SG2 & aromatic rice \\
\hline RG115 & IG 43(EC 728788- 117759) & 0.992 & 0.008 & SG1 & indica \\
\hline RG116 & RPHP 27 & 0.52 & 0.48 & $A D$ & Tropical Japonica \\
\hline RG117 & IG 65(EC 729024- 120958) & 0.974 & 0.026 & SG1 & indica \\
\hline RG118 & Ponmani samba & 0.973 & 0.027 & SG1 & indica \\
\hline RG119 & Ganthasala & 0.993 & 0.007 & SG1 & indica \\
\hline RG120 & Thattan samba & 0.949 & 0.051 & SG1 & indica \\
\hline RG121 & IG 74(EC 728622- 117517) & 0.16 & 0.84 & SG2 & japonica \\
\hline RG122 & Kaliyan samba & 0.245 & 0.755 & $A D$ & indica \\
\hline RG123 & IG 2(EC 729808-121874) & 0.56 & 0.44 & $A D$ & japonica \\
\hline RG124 & IG 29(EC 728925- 117920) & 0.059 & 0.941 & SG2 & Tropical Japonica \\
\hline RG125 & RPHP 55 & 0.963 & 0.037 & SG1 & indica \\
\hline RG126 & Kallimadayan & 0.984 & 0.016 & SG1 & indica \\
\hline RG127 & IG 10(EC 729686- 121743) & 0.066 & 0.934 & $\mathrm{SG} 2$ & aromatic \\
\hline RG128 & IG 75(EC 728587- 117420) & 0.008 & 0.992 & SG2 & japonica \\
\hline RG129 & IG 38(EC 728742 - 117707) & 0.02 & 0.98 & $\mathrm{SG} 2$ & Tropical japonica \\
\hline RG130 & IG 39(EC 728779- 117750) & 0.012 & 0.988 & $\mathrm{SG} 2$ & indica \\
\hline RG131 & RPHP 90 & 0.991 & 0.009 & SG1 & indica \\
\hline RG132 & IG 33(EC 728938- 117935) & 0.162 & 0.838 & $\mathrm{SG} 2$ & Tropical Japonica \\
\hline RG133 & IG 42(EC 728798- 117774) & 0.495 & 0.505 & $A D$ & indica \\
\hline
\end{tabular}


Table 2 Population structure group of accessions based on Inferred ancestry values (Continued)

\begin{tabular}{|c|c|c|c|c|c|}
\hline RG134 & IG 9(EC 729682- 121739) & 0.019 & 0.981 & SG2 & indica \\
\hline RG135 & RPHP 161 & 0.849 & 0.151 & SG1 & indica \\
\hline RG136 & IG 8(EC 729601- 121651) & 0.883 & 0.117 & SG1 & indica \\
\hline RG137 & IG 37(EC 728715- 117678) & 0.005 & 0.995 & SG2 & Tropical Japonica \\
\hline RG138 & Sigappu kuruvikar & 0.979 & 0.021 & SG1 & indica \\
\hline RG139 & RPHP 138 & 0.917 & 0.083 & SG1 & indica \\
\hline RG140 & Raja mannar & 0.989 & 0.011 & SG1 & indica \\
\hline RG141 & IG 44(EC 728762- 117729) & 0.134 & 0.866 & SG2 & indica \\
\hline RG142 & Sasyasree & 0.989 & 0.011 & SG1 & indica \\
\hline RG143 & IG 46(IC 471826- 117647) & 0.073 & 0.927 & SG2 & indica \\
\hline RG144 & Chetty samba & 0.993 & 0.007 & SG1 & indica \\
\hline RG145 & IG 60(EC 728730- 117695) & 0.033 & 0.967 & SG2 & indica \\
\hline RG146 & IR 75862-206 & 0.013 & 0.987 & SG2 & Tropical Japonica \\
\hline RG147 & IG 58(EC 728725- 117689) & 0.011 & 0.989 & SG2 & japonica \\
\hline RG148 & Chinna aduku nel & 0.798 & 0.202 & SG1 & indica \\
\hline RG149 & $\mathrm{RH} 2-\mathrm{SM}-2-23$ & 0.296 & 0.704 & $A D$ & indica \\
\hline RG150 & IG 14(IC 517381- 121422) & 0.775 & 0.225 & $A D$ & indica \\
\hline RG151 & IG 32(EC 728838- 117823) & 0.065 & 0.935 & SG2 & japonica \\
\hline RG152 & RPHP 47 & 0.989 & 0.011 & SG1 & indica \\
\hline RG153 & Sembilipiriyan & 0.933 & 0.067 & SG1 & indica \\
\hline RG154 & IG 48(EC 729203- 121195) & 0.006 & 0.994 & SG2 & indica \\
\hline RG155 & Sona mahsuri & 0.889 & 0.111 & SG1 & indica \\
\hline RG156 & IG 12(EC 729626- 121681) & 0.405 & 0.595 & $A D$ & indica \\
\hline RG157 & Karungan & 0.602 & 0.398 & $A D$ & indica \\
\hline RG158 & IG 13(EC 729640- 121696) & 0.143 & 0.857 & SG2 & indica \\
\hline RG159 & Sembala & 0.934 & 0.066 & SG1 & indica \\
\hline RG160 & IG 72(EC 728650- 117587) & 0.992 & 0.008 & SG1 & indica \\
\hline RG161 & Panamarasamba & 0.978 & 0.022 & SG1 & indica \\
\hline RG162 & IR 64 & 0.995 & 0.005 & SG1 & indica \\
\hline RG163 & Mikuruvai & 0.992 & 0.008 & SG1 & indica \\
\hline RG164 & Thillainayagam & 0.939 & 0.061 & SG1 & indica \\
\hline RG165 & ARB 64 & 0.843 & 0.157 & SG1 & indica \\
\hline RG166 & RPHP 140 & 0.959 & 0.041 & SG1 & indica \\
\hline RG167 & IG 70(EC 729045- 120983) & 0.989 & 0.011 & SG1 & indica \\
\hline RG168 & Haladichudi & 0.993 & 0.007 & SG1 & indica \\
\hline RG169 & IG 24(EC 728751- 117718) & 0.725 & 0.275 & $A D$ & Aus \\
\hline RG170 & RPHP 42 & 0.981 & 0.019 & SG1 & indica \\
\hline RG171 & RPHP 44 & 0.951 & 0.049 & SG1 & indica \\
\hline RG172 & IG 25(EC 729728- 121785) & 0.903 & 0.097 & SG1 & Tropical Japonica \\
\hline RG173 & IG 73(EC 728627- 117527) & 0.991 & 0.009 & SG1 & indica \\
\hline RG174 & IG 51(EC 728772- 117742) & 0.008 & 0.992 & SG2 & Tropical Japonica \\
\hline RG175 & Vellai kudaivazhai & 0.786 & 0.214 & SG1 & indica \\
\hline RG176 & Kodai & 0.906 & 0.094 & SG1 & indica \\
\hline RG177 & Kallundaikar & 0.951 & 0.049 & SG1 & indica \\
\hline RG178 & IG 17(EC 728900- 117889) & 0.993 & 0.007 & SG1 & indica \\
\hline
\end{tabular}


Table 2 Population structure group of accessions based on Inferred ancestry values (Continued)

\begin{tabular}{llllll}
\hline RG179 & Avasara samba & 0.939 & 0.061 & SG1 & indica \\
RG180 & IG 59(EC 728729- 117694) & 0.093 & 0.907 & SG2 & Tropical Japonica \\
RG181 & IG 52(EC 728756- 117723) & 0.026 & 0.974 & SG2 & Tropical Japonica \\
RG182 & ARB 59 & 0.779 & 0.221 & SG1 & indica \\
RG183 & RPHP 163 & 0.995 & 0.005 & SG1 & indica \\
RG184 & IG 18(EC 728892- 117880) & 0.994 & 0.006 & SG1 & indica \\
RG185 & RPHP 36 & 0.915 & 0.085 & SG1 & Tropical Japonica \\
RG186 & IG 28(EC 728920- 117914) & 0.009 & 0.991 & SG2 & indica \\
RG187 & Vadakathi samba & 0.986 & 0.014 & SG1 & indica \\
RG188 & RPHP 80 & 0.986 & 0.014 & SG1 & Tropical japonica \\
RG189 & IG 41(EC 728800- 117776) & 0.016 & 0.984 & SG2 & aromatic \\
RG190 & IG 26(IC 0590943- 121899) & 0.422 & 0.578 & SG2 & indica \\
RG191 & IG 15(EC 728910- 117901) & 0.755 & 0.245 & indica \\
RG192 & Nootri pathu & 0.943 & 0.057 & SG1 \\
\hline
\end{tabular}

that allowed the amplification of 7 alleles. The PIC value was found to be 0.418 for SG1 which had the majority of indica accessions. The subgroup SG2 dominated by japonica accessions had the PIC value of 0.414 . Hence, both the subgroups contribute in a major way for population diversity. As this population encompass different rice materials i.e., landraces, varieties and breeding lines, the molecular diversity is contributed majorly by landraces. These values are similar to those found by Courtois et al. (2012) who reported the PIC value from 0.16 to 0.78 with the average of 0.49 in European rice germplasm collection and in Chinese rice collection of 416 accessions by Jin et al. (2010), who has given similar PIC value of 0.4214 . It is also consistent with PIC value (0.48) attained by Zhang et al. (2011). In this study, significant amount of rare alleles was identified which indicates that these rare alleles contribute well to the overall genetic diversity of the population.

Model based approach by STRUCTURE is implemented frequently for studying population structure by various researchers (Agrama et al. 2007, Agrama and Eizenga 2008; Garris et al. 2005; Zhang et al. 2007, 2011; Jin et al. 2010; Liakat Ali et al. 2011, Chakhonkaen et al. 2012 Courtois et al. 2012, Das et al. 2013). Courtois et al. (2012) has successfully detected two subgroups in their study population and assigned rice varieties into two groups with few admixture lines. Jin et al. (2010) has identified seven sub populations among 416 rice accessions from China. Das et al. (2013) has grouped a collection of 91 accessions of rice landraces from eastern and north eastern India into four groups.

Assigning of genotypes to the subgroups based on ancestry threshold vary between different research groups.

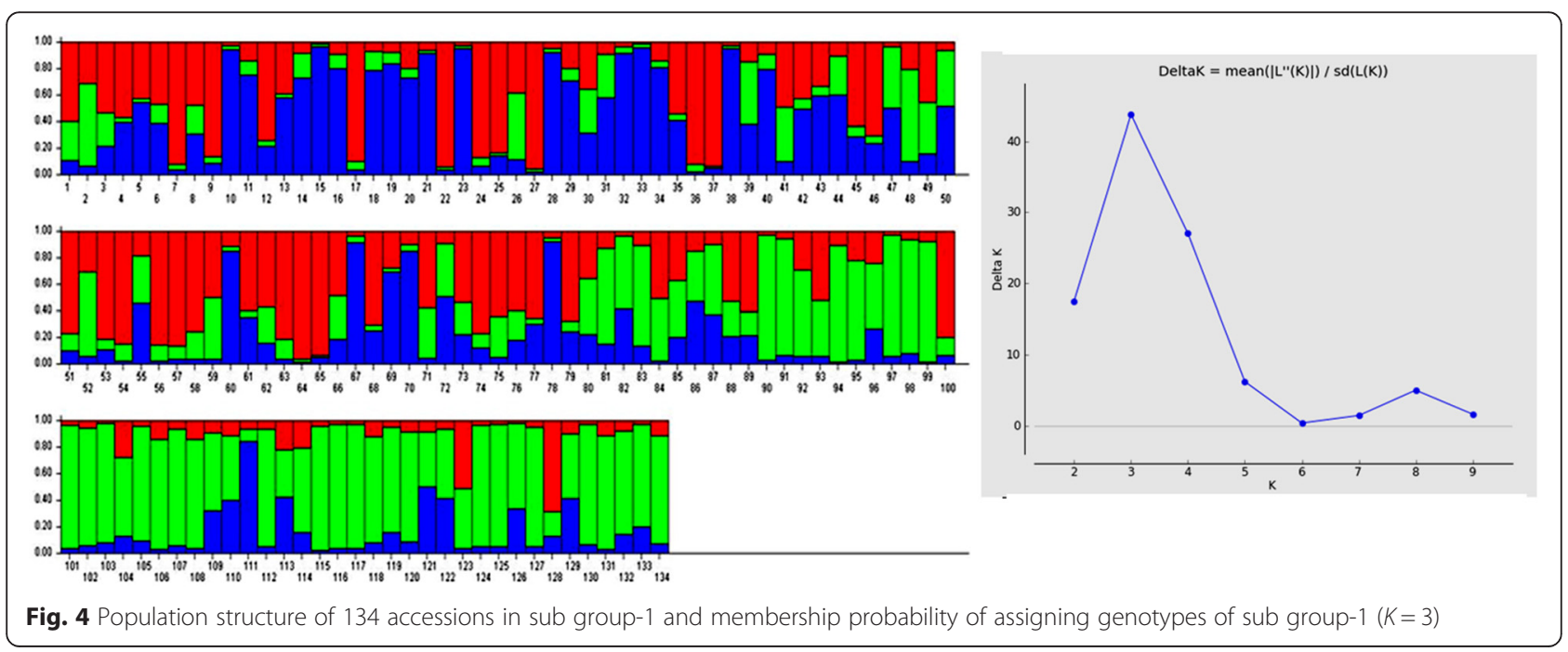




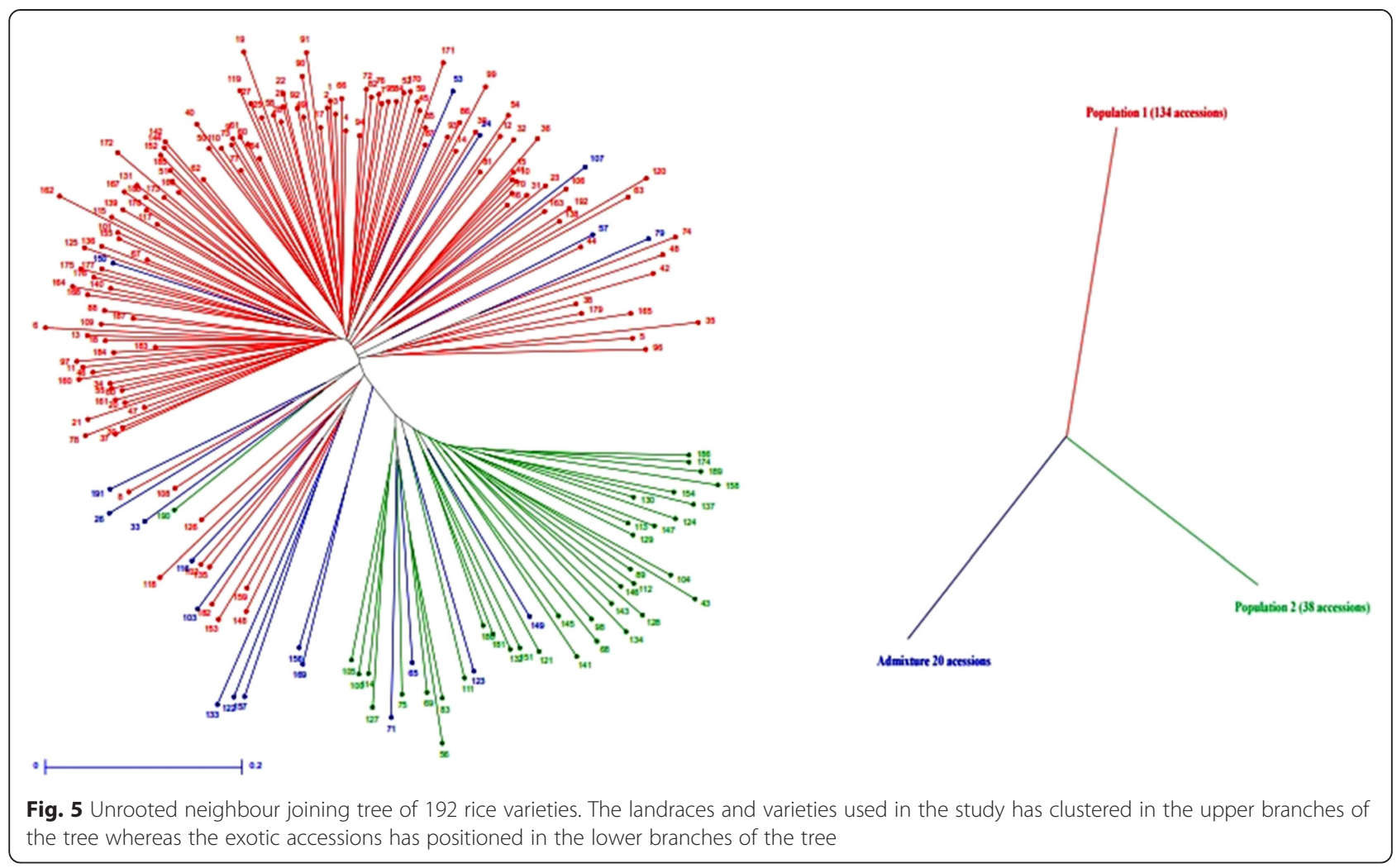

Zhao et al. (2010) and Courtois et al. (2012) used an ancestry threshold of $80 \%$ to identify accessions belonging to a specific subpopulation. Liakat Ali et al. (2011) has steup the threshold as $60 \%$ and identified 33 accessions as admixtures as the threshold of $80 \%$ consider more genotypes as admixtures. In the current study, a stringent threshold of $80 \%$ ancestry value leaves only 20 genotypes as admixtures.

Population structure analysis in different rice diversity panel has indicated the existence of two to eight sub population in rice (Zhang et al. 2007, Zhang et al. 2009, Zhang et al. 2011, Garris et al. 2005, Agrama et al. 2007, Liakat Ali et al. 2011, Chakhonkaen et al. 2012 and Das et al. 2013). In the current rice diversity panel of 192 accessions based on the criterion of maximum membership probabilities, 134 accessions were assigned to SG1 which is dominated by indica subtype with most of the landraces and varieties of Indian origin and SG2 consisted of 38 accessions which composed mostly of japonica accessions of exotic origin. Similar population structure of two subgroups was observed in previous research by Zhang et al. (2009) in a collection of 3024 rice landraces in China. Zhang et al. (2011) has reported two distinct subgroups in a rice core collection. Courtois et al. (2012) has successfully classified two subgroups as japonica and non japonica accessions in European core collection of rice. The results indicated that two subgroups are due to the different adaptation behavior of accessions to different ecological environment as indica and japonica accessions has independent evolution frame and the origin of Indian rice accessions from indica cultivars. Hence the major criterion for population structure in this panel is indica - japonica subtype. This study includes large number of traditional landraces and varieties from Indian Subcontinent and few exotic accessions randomly selected from IRRI worldwide collection. It clarifies the relationship between Indian germplasm and exotic accessions which indicates that germplasm lines varies based on its ecology and also shows higher level of genetic diversity exists within this population.

Further structure analysis of SG1 that consisted of 134 lines indicated that it can be further subdivided in to three sub sub-groups. The three sub sub-groups classification has the factor of ecosystem and seasonal variation as the major factors for population structure. This results is in accordance with the inference that indica group has higher genetic diversity than japonica accessions which was given by various researchers (Gao et al. 2005; Lu et al. 2005; Lapitan et al. 2007; Caicedo et al. 2007; Liakat Ali et al. 2011; Garris et al. 2005; Qi et al. 2006; Qi et al. 2009); as this subgroup has indica accessions. Liakat Ali et al. (2011) has substantiated this statement with the reason of the indica subpopulation occupying the largest rice growing region which has a varied environments, ecological conditions and soil type. 


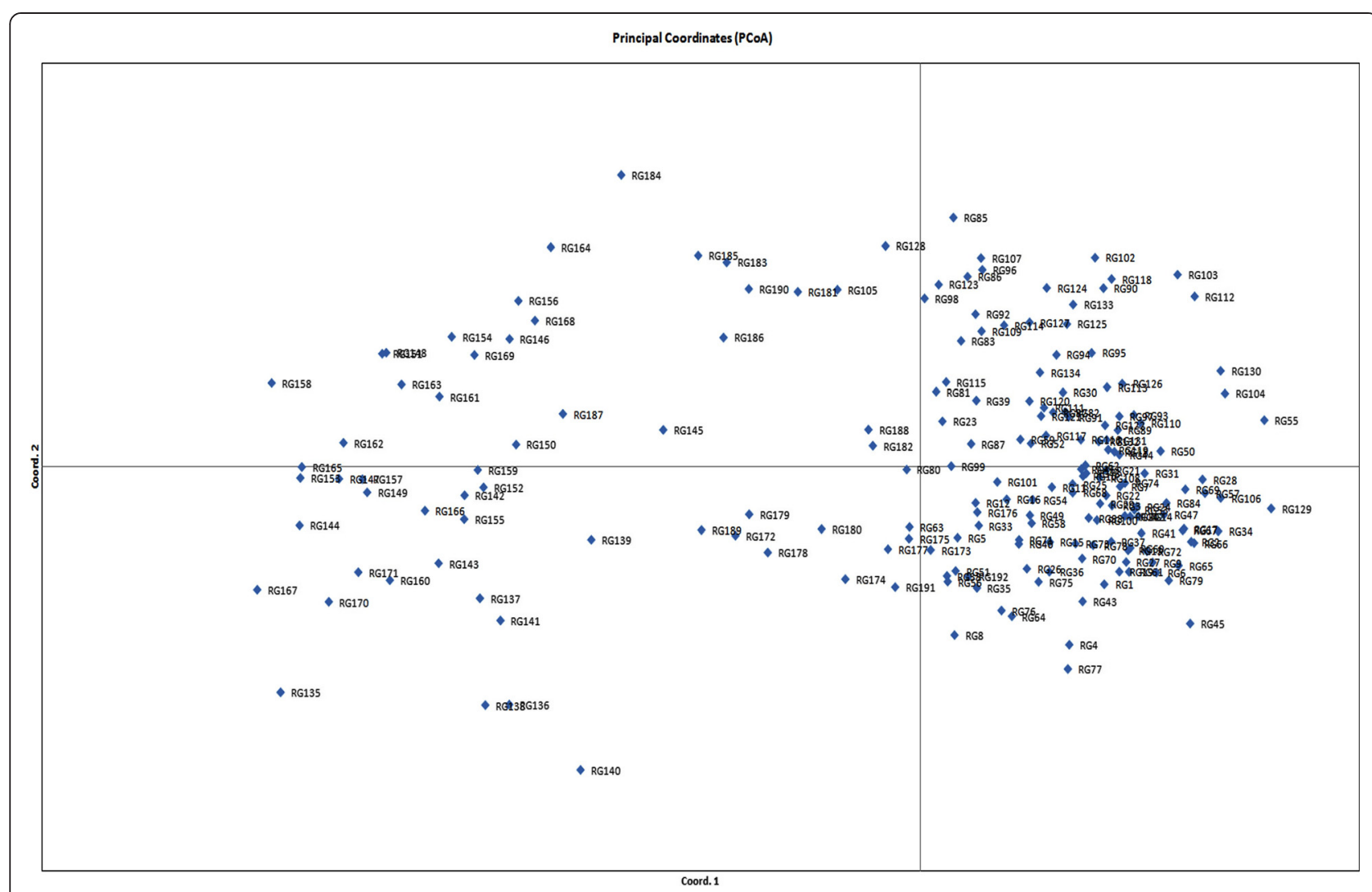

Fig. 6 Principal Coordinates of 192 accessions based on 61 SSR loci. Coord 1 and Coord 2 represent first and second coordinates, respectively. The two PCA axes accounted for 12.6 and $4.9 \%$ of the genetic variation among populations

The result of model based analysis is in accordance with the clustering pattern of Neighbour joining tree and Principal Coordinate Analysis. The first two principal coordinates explained 12.6 and $4.8 \%$ of the molecular variance. Similar pattern of molecular variance explanation was observed by Zhang et al. (2011) for two population subgroups.

Calculation of Wright's F Statistic at all loci revealed the deviation from Hardy- Weinberg law for molecular variation within the population. The result of $F_{s t}$

Table 3 AMOVA between groups and Pair wise comparison using Fst values (GenAlEx)

\begin{tabular}{llrrrl}
\hline Source & df & \multicolumn{1}{l}{ SS } & MS & Est. var. & Percent \\
\hline Among the population & 2 & 971.922 & 485.961 & 9.631 & $14 \%$ \\
Within Pops & 189 & 10961.256 & 57.996 & 57.996 & $86 \%$ \\
Total & 191 & 11933.177 & & 67.627 & $100 \%$
\end{tabular}

Pairwise population Fst values

\begin{tabular}{lll} 
& SG2 & AD \\
SG1 & 0.128 & 0.040 \\
SG2 & & 0.061 \\
\hline
\end{tabular}

indicates higher divergence existing between subgroups of the population. Higher $\mathrm{F}_{\mathrm{IT}}$, which is measured at subgroup level in whole population, has indicated lack of equilibrium across the groups and lack of heterozygosity most likely due to the inbreeding nature of rice.

The present study revealed that several unexploited landraces of Tamil Nadu, India which is widely cultivated

Table 4 AMOVA between groups and accessions and Fixation indices (Arlequin software)

\begin{tabular}{llccc}
\hline Source of variation & d.f. & $\begin{array}{c}\text { Sum of } \\
\text { squares }\end{array}$ & $\begin{array}{l}\text { Variance } \\
\text { components }\end{array}$ & $\begin{array}{c}\text { Percentage } \\
\text { of variation }\end{array}$ \\
\hline Among Populations & 2 & 200.013 & $1.01840 \mathrm{Va}$ & 13.82 \\
$\begin{array}{l}\text { Among individuals } \\
\text { within Populations }\end{array}$ & 189 & 1794.771 & $3.14391 \mathrm{Vb}$ & 42.65 \\
Within Individuals & 192 & 616 & $3.20833 \mathrm{Vc}$ & 43.53 \\
& 383 & 2610.784 & 7.37064 & \\
Fixation Indices & & & & \\
FIS & 0.49493 & & \\
FST & 0.13817 & & \\
FIT & 0.56471 & & & \\
\hline
\end{tabular}


Table 5 Genotypes selected for association mapping panel

\begin{tabular}{|c|c|c|c|c|c|c|c|c|c|c|c|}
\hline G. no & Genotypes & G. no & Genotypes & G. no & Genotypes & G. no & Genotypes & G. no & Genotypes & G. no & Genotypes \\
\hline RG1 & $\begin{array}{l}\text { Mapillai } \\
\text { samba }\end{array}$ & RG58 & Kodaikuluthan & RG113 & IG 45(EC 728768- 117736) & RG154 & IG 48(EC 729203- 121195) & RG39 & Kaatu ponni & RG95 & Jeeraga samba \\
\hline RG2 & CK 275 & RG59 & RPHP 68 & RG114 & RPHP 159 & RG156 & IG 12(EC 729626- 121681) & RG41 & Godavari samba & RG96 & RP-BIO-226 \\
\hline RG3 & Senkar & RG60 & Rama kuruvaikar & RG115 & IG 43(EC 728788- 117759) & RG157 & Karungan & RG42 & Earapalli samba & RG98 & IG 5(EC 729642- 121698) \\
\hline RG4 & Murugankar & RG62 & Purple puttu & RG116 & RPHP 27 & RG158 & IG 13(EC 729640- 121696) & RG43 & RPHP 129 & RG99 & IG 31(EC 728844- 117829) \\
\hline RG5 & CHIR 6 & RG63 & IG 71(EC 728651- 117588) & RG117 & IG 65(EC 729024- 120958) & RG159 & Sembala & RG44 & Mangam samba & RG100 & IG 7(EC 729598- 121648) \\
\hline RG6 & CHIR 5 & RG65 & IG 56(EC 728700- 117658 & RG118 & Ponmani samba & RG160 & IG 72(EC 728650- 117587) & RG45 & RPHP 105 & RG101 & RPHP 52 \\
\hline RG7 & Kudai vazhai & RG66 & Jeevan samba & RG120 & Thattan samba & RG161 & Panamarasamba & RG46 & IG 4(EC 729639- 121695) & RG102 & Varakkal \\
\hline RG8 & CHIR 8 & RG67 & RPHP 106 & RG121 & IG 74(EC 728622- 117517) & RG162 & IR 64 & RG48 & Kalarkar & RG103 & Mattaikar \\
\hline RG9 & $\begin{array}{l}\text { Kuruvai } \\
\text { kalanjiyam }\end{array}$ & RG68 & IG 63(EC 728711- 117674) & RG122 & Kaliyan samba & RG163 & Mikuruvai & RG50 & Sornavari & RG104 & IG 53(EC 728752- 117719) \\
\hline RG12 & $\begin{array}{l}\text { Vellai } \\
\text { chithiraikar }\end{array}$ & RG69 & RPHP 48 & RG123 & IG 2(EC 729808-121874) & RG164 & Thillainayagam & RG51 & RPHP 134 & RG105 & IG 6(EC 729592- 121642) \\
\hline RG14 & Jothi & RG70 & Karthi samba & RG124 & IG 29(EC 728925- 117920) & RG165 & ARB 64 & RG52 & ARB 58 & RG106 & Katta samba \\
\hline RG15 & Palkachaka & RG71 & IG 27(IC 0590934- 121255) & RG126 & Kallimadayan & RG166 & RPHP 140 & RG53 & IR 68144-2B-2-2-3-1-127 & RG107 & $\mathrm{RH} 2-\mathrm{SM}-1-2-1$ \\
\hline RG17 & $\begin{array}{l}\text { Chivapu } \\
\text { chithiraikar }\end{array}$ & RG72 & Aarkadu kichili & RG127 & IG 10(EC 729686- 121743) & RG168 & Haladichudi & RG54 & PTB 19 & RG108 & Red sirumani \\
\hline RG18 & CHIR 11 & RG74 & ARB 65 & RG128 & IG 75(EC 728587- 117420) & RG169 & IG 24(EC 728751- 117718) & RG55 & IG 67(EC 729050- 120988) & RG109 & Vadivel \\
\hline RG20 & Kalvalai & RG76 & Matta kuruvai & RG129 & IG 38(EC 728742 - 117707) & RG170 & RPHP 42 & RG56 & RPHP 59 & RG110 & Norungan \\
\hline RG22 & IR 36 & RG77 & Karuthakar & RG130 & IG 39(EC 728779- 117750) & RG172 & IG 25(EC 729728- 121785) & RG57 & RPHP 103 & RG112 & IG 35(EC 728858- 117843) \\
\hline RG25 & Sorna kuruvai & RG80 & IG 66(EC 729047- 120985) & RG131 & RPHP 90 & RG173 & IG 73(EC 728627- 117527) & RG143 & IG 46(IC 471826- 117647) & RG184 & IG 18(EC 728892- 117880) \\
\hline RG26 & Rascadam & RG81 & CB-07-701-252 & RG132 & IG 33(EC 728938- 117935) & RG174 & IG 51(EC 728772- 117742) & RG145 & IG 60(EC 728730- 117695) & RG185 & RPHP 36 \\
\hline RG31 & Chinthamani & RG82 & Thooyamalli & RG133 & IG 42(EC 728798- 117774) & RG175 & Vellai kudaivazhai & RG146 & IR 75862-206 & RG186 & IG 28(EC 728920- 117914) \\
\hline RG32 & Thogai samba & RG83 & RPHP 93 & RG134 & IG 9(EC 729682- 121739) & RG176 & Kodai & RG147 & IG 58(EC 728725- 117689) & RG187 & Vadakathi samba \\
\hline RG33 & $\begin{array}{l}\text { Malayalathan } \\
\text { samba }\end{array}$ & RG85 & RPHP 104 & RG135 & RPHP 161 & RG178 & IG 17(EC 728900- 117889) & RG148 & Chinna aduku nel & RG188 & RPHP 80 \\
\hline RG34 & RPHP 125 & RG86 & RPHP 102 & RG136 & IG 8(EC 729601- 121651) & RG180 & IG 59(EC 728729- 117694) & RG149 & $\mathrm{RH} 2-\mathrm{SM}-2-23$ & RG189 & IG 41(EC 728800- 117776) \\
\hline RG35 & CK 143 & RG89 & IR 83294-66-2-2-3-2 & RG137 & IG 37(EC 728715- 117678) & RG181 & IG 52(EC 728756- 117723) & RG150 & IG 14(IC 517381- 121422) & RG190 & IG 26(IC 0590943- 121899) \\
\hline RG36 & Kattikar & RG91 & IG 23(EC 729391- 121419) & RG141 & IG 44(EC 728762- 117729) & RG182 & ARB 59 & RG151 & IG 32(EC 728838- 117823) & RG191 & IG 15(EC 728910- 117901) \\
\hline RG37 & Shenmolagai & RG92 & IG 49(EC 729102- 121052) & RG142 & Sasyasree & RG183 & RPHP 163 & RG152 & RPHP 47 & RG192 & Nootri pathu \\
\hline
\end{tabular}


Table 6 Germplasm accessions used in the study

\begin{tabular}{|c|c|c|c|c|c|c|c|c|}
\hline G. no. & Genotype & Parentage & Origin & $\begin{array}{l}\text { Type - } \\
\text { traditional/ } \\
\text { Improved }\end{array}$ & Subtype & $\begin{array}{l}\text { Ecosystem } I R=\text { irrigated } \\
R L=\text { rainfed lowland; } \\
U P=\text { upland }\end{array}$ & $\begin{array}{l}\text { Maturity class: } \mathrm{E}=\text { early, } \\
\mathrm{M}=\text { medium, } \mathrm{L}=\text { late; }\end{array}$ & $\begin{array}{l}\text { Donors/Original } \\
\text { providing country }\end{array}$ \\
\hline$\overline{R G 1}$ & Mapillai samba & Landrace & Tamil Nadu, India & $T$ & indica & $\mathbb{I R}$ & $\mathrm{L}$ & India \\
\hline RG2 & CK 275 & CO50 X KAVUNI & Tamil Nadu, India & I & indica & $\mathbb{R}$ & $\mathrm{L}$ & India \\
\hline RG3 & Senkar & Landrace & Tamil Nadu, India & $\mathrm{T}$ & indica & $\mathbb{R}$ & M & India \\
\hline RG4 & Murugankar & Landrace & Tamil Nadu, India & $\mathrm{T}$ & indica & UP & L & India \\
\hline RG5 & $\mathrm{CHIR} 6$ & Improved chinsurah & West Bengal & । & indica & $\mathbb{R}$ & $\mathrm{E}$ & India \\
\hline RG6 & CHIR 5 & Improved chinsurah & West Bengal & । & indica & $\mathbb{R}$ & $\mathrm{E}$ & India \\
\hline RG7 & Kudai vazhai & Landrace & Tamil Nadu, India & $\mathrm{T}$ & indica & UP & $\mathrm{E}$ & India \\
\hline RG8 & CHIR 8 & Improved chinsurah & West Bengal & । & indica & $\mathbb{R}$ & $\mathrm{E}$ & India \\
\hline RG9 & Kuruvai kalanjiyam & Landrace & Tamil Nadu, India & $\mathrm{T}$ & indica & $\mathbb{R}$ & $E$ & India \\
\hline RG10 & Nava konmani & Landrace & Tamil Nadu, India & $\mathrm{T}$ & indica & $\mathrm{RL}$ & M & India \\
\hline RG11 & CHIR 10 & Improved chinsurah & West Bengal & I & indica & $\mathbb{R}$ & M & India \\
\hline RG12 & Vellai chithiraikar & Landrace & Tamil Nadu, India & $\mathrm{T}$ & indica & $\mathrm{RL}$ & $\mathrm{E}$ & India \\
\hline RG13 & $\mathrm{CHIR} 2$ & Improved chinsurah & West Bengal & I & indica & $\mathbb{R}$ & M & India \\
\hline RG14 & Jyothi & Variety & Kerala, India & $\mathrm{T}$ & indica & $\mathbb{R}$ & E & India \\
\hline RG15 & Palkachaka & Landrace & Tamil Nadu, India & $\mathrm{T}$ & indica & $\mathbb{R}$ & M & India \\
\hline RG16 & Thooyala & Landrace & Tamil Nadu, India & $\mathrm{T}$ & indica & $\mathbb{R}$ & $\mathrm{E}$ & India \\
\hline RG17 & Chivapu chithiraikar & Landrace & Tamil Nadu, India & $\mathrm{T}$ & indica & $\mathrm{RL}$ & $\mathrm{E}$ & India \\
\hline RG18 & CHIR 11 & Improved chinsurah & West Bengal & । & indica & $\mathbb{R}$ & M & India \\
\hline RG19 & Koolavalai & Landrace & Tamil Nadu, India & $\mathrm{T}$ & indica & $\mathrm{RL}$ & M & India \\
\hline RG20 & Kalvalai & Landrace & Tamil Nadu, India & $\mathrm{T}$ & indica & $\mathrm{RL}$ & $\mathrm{E}$ & India \\
\hline RG21 & Mohini samba & Landrace & Tamil Nadu, India & $\mathrm{T}$ & indica & $\mathbb{R}$ & M & India \\
\hline $\mathrm{RG} 22$ & IR 36 & $\begin{array}{l}\text { IR } 1561 \text { X IR } 24 \text { X Oryza } \\
\text { nivara x CR } 94\end{array}$ & IRRI, Philippines & I & indica & $\mathbb{I R}$ & $\mathrm{E}$ & Philippines \\
\hline RG23 & Koombalai & Landrace & Tamil Nadu, India & $\mathrm{T}$ & indica & $\mathbb{R}$ & M & India \\
\hline RG24 & Tadukan & Landrace & Philippines & $\mathrm{T}$ & indica & UP & M & Philippines \\
\hline RG25 & Sorna kuruvai & Landrace & Tamil Nadu, India & $\mathrm{T}$ & indica & $\mathbb{I R}$ & M & India \\
\hline RG26 & Rascadam & Landrace & Tamil Nadu, India & $\mathrm{T}$ & indica & $\mathbb{R}$ & M & India \\
\hline RG27 & Muzhi karuppan & Landrace & Tamil Nadu, India & $\mathrm{T}$ & indica & $\mathbb{R}$ & $\mathrm{E}$ & India \\
\hline$R G 28$ & Kaatukuthalam & Landrace & Tamil Nadu, India & $\mathrm{T}$ & indica & $\mathrm{RL}$ & M & India \\
\hline RG29 & Vellaikattai & Landrace & Tamil Nadu, India & $\mathrm{T}$ & indica & $\mathrm{RL}$ & M & India \\
\hline RG30 & Poongar & Landrace & Tamil Nadu, India & $\mathrm{T}$ & indica & $R L$ & L & India \\
\hline
\end{tabular}


Table 6 Germplasm accessions used in the study (Continued)

\begin{tabular}{|c|c|c|c|c|c|c|c|c|}
\hline RG31 & Chinthamani & Landrace & Tamil Nadu, India & $\mathrm{T}$ & indica & $\mathrm{RL}$ & M & India \\
\hline RG32 & Thogai samba & Landrace & Tamil Nadu, India & $\mathrm{T}$ & indica & $\mathrm{RL}$ & M & India \\
\hline RG33 & Malayalathan samba & Landrace & Tamil Nadu, India & $\mathrm{T}$ & indica & $\mathbb{R}$ & E & India \\
\hline RG34 & RPHP125 & NDR 2026 (RICHA) & UTTAR PRADHESH & I & indica & $\mathbb{R}$ & $E$ & India \\
\hline RG35 & CK 143 & CO50 X KAVUNI & Tamil Nadu, India & I & indica & $\mathbb{R}$ & L & India \\
\hline RG36 & Kattikar & Landrace & Tamil Nadu, India & $\mathrm{T}$ & indica & $\mathrm{RL}$ & M & India \\
\hline RG37 & Shenmolagai & Landrace & Tamil Nadu, India & $\mathrm{T}$ & indica & $\mathbb{R}$ & M & India \\
\hline RG38 & Velli samba & Landrace & Tamil Nadu, India & $\mathrm{T}$ & indica & $\mathbb{R}$ & M & India \\
\hline RG39 & Kaatu ponni & Landrace & Tamil Nadu, India & $\mathrm{T}$ & indica & $\mathbb{R}$ & M & India \\
\hline RG40 & kakarathan & Landrace & Tamil Nadu, India & $\mathrm{T}$ & indica & $\mathbb{I R}$ & M & India \\
\hline RG41 & Godavari samba & Landrace & Tamil Nadu, India & $\mathrm{T}$ & indica & $\mathbb{R}$ & M & India \\
\hline RG42 & Earapalli samba & Landrace & Tamil Nadu, India & $\mathrm{T}$ & indica & $\mathbb{R}$ & M & India \\
\hline RG43 & RPHP 129 & Kamad & JAMMU \& KASHMIR & $\mathrm{T}$ & indica & Scented & E & India \\
\hline RG44 & Mangam samba & Landrace & Tamil Nadu, India & $\mathrm{T}$ & indica & $\mathbb{R}$ & M & India \\
\hline RG45 & RPHP 105 & Moirang phou & MANIPUR & $\mathrm{T}$ & indica & $\mathbb{R}$ & E & India \\
\hline RG46 & IG 4(EC 729639- 121695) & TD2: :IRGC 9148-1 & IRRI, Philippines & । & indica & $\mathbb{R}$ & M & Philippines \\
\hline RG47 & Machakantha & Landrace & Orissa, India & $\mathrm{T}$ & indica & scented & E & India \\
\hline RG48 & Kalarkar & Landrace & Tamil Nadu, India & $\mathrm{T}$ & indica & $\mathrm{RL}$ & E & India \\
\hline RG49 & Valanchennai & Landrace & Tamil Nadu, India & $\mathrm{T}$ & indica & $\mathrm{RL}$ & E & India \\
\hline RG50 & Sornavari & Landrace & Tamil Nadu, India & $\mathrm{T}$ & indica & $\mathrm{RL}$ & $E$ & India \\
\hline RG51 & RPHP 134 & NJAVARA & Kerala & $\mathrm{T}$ & indica & RL & E & India \\
\hline RG52 & ARB 58 & Variety & Karnataka & 1 & indica & $\mathbb{R}$ & E & India \\
\hline RG53 & IR 68144-2B-2-2-3-1-127 & IR 72 X ZAWA BONDAY & IRRI, Philippines & 1 & indica & & E & Philippines \\
\hline RG54 & PTB 19 & Variety & Kerala, India & I & indica & $\mathbb{R}$ & M & India \\
\hline RG55 & IG 67(EC 729050- 120988) & $\begin{array}{l}\text { IR 77384-12-35-3-12-I-B::IRGC } \\
\text { 117299-1 }\end{array}$ & IRRI, Philippines & I & indica & $\mathbb{R}$ & E & Philippines \\
\hline RG56 & RPHP 59 & Taroari Basmati/karnal local & HARYANA & $\mathrm{T}$ & Aromatic & scented & $L$ & India \\
\hline RG57 & RPHP 103 & Pant sugandh dhan -17 & UTTARKHAND & I & Aromatic & scented & L & India \\
\hline RG58 & Kodaikuluthan & Landrace & Tamil Nadu, India & $\mathrm{T}$ & indica & $\mathrm{RL}$ & E & India \\
\hline RG59 & RPHP 68 & Subhdra & Orissa, India & 1 & indica & $\mathrm{RL}$ & $E$ & India \\
\hline RG60 & Rama kuruvaikar & Landrace & Tamil Nadu, India & $\mathrm{T}$ & indica & $\mathbb{I R}$ & $E$ & India \\
\hline RG61 & Kallundai & Landrace & Tamil Nadu, India & $\mathrm{T}$ & indica & $\mathrm{RL}$ & E & India \\
\hline RG62 & Purple puttu & Landrace & Tamil Nadu, India & $\mathrm{T}$ & indica & $\mathbb{I R}$ & E & India \\
\hline
\end{tabular}


Table 6 Germplasm accessions used in the study (Continued)

\begin{tabular}{|c|c|c|c|c|c|c|c|c|}
\hline RG63 & IG 71(EC 728651- 117588) & TEPI BORO::IRGC 27519-1 & IRRI, Philippines & 1 & aus & $\mathrm{IR}$ & $E$ & Philippines \\
\hline RG64 & Ottadaiyan & Landrace & Tamil Nadu, India & $\mathrm{T}$ & indica & $\mathrm{RL}$ & M & India \\
\hline RG65 & IG 56(EC 728700- 117658 & BICO BRANCO & Brazil & $\mathrm{T}$ & Aromatic & UP & $\mathrm{E}$ & Philippines \\
\hline RG66 & Jeevan samba & Landrace & Tamil Nadu, India & $\mathrm{T}$ & indica & $\mathbb{R}$ & M & India \\
\hline RG67 & RPHP 106 & akut phou & MANIPUR & । & indica & $\mathbb{R}$ & M & India \\
\hline RG68 & IG 63(EC 728711- 117674) & CAAWA/FORTUNA & IRRI, Philippines & । & $\begin{array}{l}\text { Tropical } \\
\text { Japonica }\end{array}$ & $\mathbb{R}$ & M & Philippines \\
\hline RG69 & RPHP 48 & Bindli & UTTARKHAND & $\mathrm{T}$ & Aromatic & Scented & $\mathrm{L}$ & India \\
\hline RG70 & Karthi samba & Landrace & Tamil Nadu, India & $\mathrm{T}$ & indica & $\mathbb{R}$ & M & India \\
\hline RG71 & IG 27(IC 0590934- 121255) & ARC 11345::IRGC 21336-1 & IRRI, Philippines & । & indica & $\mathbb{R}$ & M & Philippines \\
\hline RG72 & Aarkadu kichili & Landrace & Tamil Nadu, India & $\mathrm{T}$ & indica & $\mathbb{R}$ & M & India \\
\hline RG73 & Kunthali & Landrace & Tamil Nadu, India & $\mathrm{T}$ & indica & $\mathbb{R}$ & $\mathrm{E}$ & India \\
\hline RG74 & ARB 65 & Variety & Karnataka & । & indica & $\mathbb{R}$ & $\mathrm{E}$ & India \\
\hline RG75 & IG 21(EC 729334- 121355) & HONGJEONG::IRGC 73052-1 & IRRI, Philippines & । & japonica & $\mathbb{R}$ & $\mathrm{E}$ & Philippines \\
\hline RG76 & Matta kuruvai & Landrace & Tamil Nadu, India & $\mathrm{T}$ & indica & $\mathbb{R}$ & $\mathrm{E}$ & India \\
\hline RG77 & Karuthakar & Landrace & Tamil Nadu, India & $\mathrm{T}$ & indica & $\mathrm{RL}$ & $\mathrm{E}$ & India \\
\hline RG78 & RPHP 165 & Tilak kachari & West Bengal & $\mathrm{T}$ & indica & $\mathbb{R}$ & $\mathrm{E}$ & India \\
\hline RG79 & Manavari & Landrace & Tamil Nadu, India & $\mathrm{T}$ & indica & U & $\mathrm{E}$ & India \\
\hline RG80 & IG 66(EC 729047- 120985) & $\begin{array}{l}\text { IR 71137-243-2-2-3-3:.IRGC } \\
\text { 99696-1 }\end{array}$ & IRRI, Philippines & । & indica & $\mathbb{I R}$ & $\mathrm{E}$ & Philippines \\
\hline RG81 & CB-07-701-252 & White ponni X Rasi & Tamil Nadu, India & । & indica & $\mathbb{R}$ & $E$ & India \\
\hline RG82 & Thooyamalli & Landrace & Tamil Nadu, India & $\mathrm{T}$ & indica & $\mathbb{R}$ & M & India \\
\hline RG83 & RPHP 93 & Type-3 (Dehradooni Basmati) & UTTARKHAND & । & indica & Scented & M & India \\
\hline RG84 & Velsamba & Landrace & Tamil Nadu, India & $\mathrm{T}$ & indica & $\mathbb{R}$ & M & India \\
\hline RG85 & RPHP 104 & Kasturi (IET 8580) & UTTARKHAND & । & indica & $\mathbb{R}$ & M & India \\
\hline RG86 & RPHP 102 & Kanchana & Kerala, India & । & indica & Semi Deep Water & $\mathrm{L}$ & India \\
\hline RG87 & IG 40(EC 728740- 117705) & DEE GEO WOO GEN & TAIWAN & $\mathrm{T}$ & Indica & $\mathbb{R}$ & M & Philippines \\
\hline RG88 & Saranga & Landrace & Tamil Nadu, India & $\mathrm{T}$ & indica & $\mathbb{R}$ & E & India \\
\hline RG89 & IR 83294-66-2-2-3-2 & DAESANBYEO X IR65564-44-5-1 & IRRI, Philippines & । & japonica & $\mathrm{RL}$ & M & Philippines \\
\hline RG90 & IG 61(EC 728731- 117696) & CRIOLLO LA FRIA & Venezuela & । & Indica & $\mathbb{R}$ & E & Philippines \\
\hline RG91 & IG 23(EC 729391- 121419) & MAHA PANNITHI::IRGC 51021-1 & IRRI, Philippines & । & Aus & $\mathbb{R}$ & M & Philippines \\
\hline RG92 & IG 49(EC 729102- 121052) & MENAKELY ::IRGC 69963-1 & Madagascar & । & Indica & $\mathrm{RL}$ & M & Philippines \\
\hline RG93 & Uppumolagai & Landrace & Tamil Nadu, India & $\mathrm{T}$ & Indica & $\mathbb{R}$ & M & India \\
\hline RG94 & Karthigai samba & Landrace & Tamil Nadu, India & $\mathrm{T}$ & Indica & $\mathrm{RL}$ & M & India \\
\hline
\end{tabular}


Table 6 Germplasm accessions used in the study (Continued)

\begin{tabular}{|c|c|c|c|c|c|c|c|c|}
\hline RG95 & Jeeraga samba & Landrace & Tamil Nadu, India & $\mathrm{T}$ & Indica & IR & M & India \\
\hline RG96 & $\mathrm{RP}-\mathrm{BIO}-226$ & IMPROVED SAMBHA MAHSURI & ANDHRA PRADESH & I & Indica & $\mathbb{R}$ & M & India \\
\hline RG97 & Varigarudan samba & Landrace & Tamil Nadu, India & $\mathrm{T}$ & Indica & $\mathbb{R}$ & M & India \\
\hline RG98 & IG 5(EC 729642- 121698) & IR 65907-116-1-B::C1 & IRRI, Philippines & । & japonica & UP & $\mathrm{E}$ & Philippines \\
\hline RG99 & IG 31(EC 728844- 117829) & ORYZICA LLANOS 5 & Colombia & $\mathrm{T}$ & Indica & $\mathbb{R}$ & M & Philippines \\
\hline RG100 & IG 7(EC 729598- 121648) & VARY MAINTY:IIRGC 69910-1 & Madagascar & । & japonica & $\mathbb{R}$ & M & Philippines \\
\hline RG101 & RPHP 52 & SEBATI & Orissa, India & I & Indica & $\mathbb{R}$ & M & India \\
\hline RG102 & Varakkal & Landrace & Tamil Nadu, India & $\mathrm{T}$ & Indica & UP & $E$ & India \\
\hline RG103 & Mattaikar & Landrace & Tamil Nadu, India & $\mathrm{T}$ & Indica & $R L$ & $L$ & India \\
\hline RG104 & IG 53(EC 728752- 117719) & CAROLINA RINALDO BARSANI & URUGUAY & 1 & $\begin{array}{l}\text { Temperate } \\
\text { japonica }\end{array}$ & $\mathbb{I R}$ & E & Philippines \\
\hline RG105 & IG 6(EC 729592- 121642) & SOM CAU 70 A:IRGC 8227-1 & Vietnam & I & $\begin{array}{l}\text { Temperate } \\
\text { japonica }\end{array}$ & $\mathbb{R}$ & E & Philippines \\
\hline RG106 & Katta samba & Landrace & Tamil Nadu, India & $\mathrm{T}$ & Indica & $R L$ & $\mathrm{~L}$ & India \\
\hline RG107 & $\mathrm{RH} 2-\mathrm{SM}-1-2-1$ & SWARNA X MOROBERAKAN & Tamil Nadu, India & 1 & Indica & $\mathbb{R}$ & $E$ & India \\
\hline RG108 & Red sirumani & Landrace & Tamil Nadu, India & $\mathrm{T}$ & Indica & $R L$ & $\mathrm{E}$ & India \\
\hline RG109 & Vadivel & Landrace & Tamil Nadu, India & $\mathrm{T}$ & Indica & $\mathbb{R}$ & M & India \\
\hline RG110 & Norungan & Landrace & Tamil Nadu, India & $T$ & Indica & $R L$ & $\mathrm{E}$ & India \\
\hline RG111 & IG 20(EC 729293- 121310) & CHIGYUNGDO:IRGC 55466-1 & South Korea & I & Indica & UP & $\mathrm{E}$ & Philippines \\
\hline RG112 & IG 35(EC 728858- 117843) & PATE BLANC MN 1 & Cote D'Ivoire & । & japonica & UP & M & Philippines \\
\hline RG113 & IG 45(EC 728768- 117736) & FORTUNA & Puerto Rico & $\mathrm{T}$ & japonica & $\mathbb{R}$ & M & Philippines \\
\hline RG114 & RPHP 159 & Radhuni Pagal & BANGLADESH & 1 & aromatic rice & Scented & $\mathrm{L}$ & India \\
\hline RG115 & IG 43(EC 728788- 117759) & IR-44595 & IRRI, Philippines & । & indica & $\mathbb{R}$ & $\mathrm{E}$ & Philippines \\
\hline RG116 & RPHP 27 & Azucena & IRRI, Philippines & $\mathrm{T}$ & $\begin{array}{l}\text { Tropical } \\
\text { Japonica }\end{array}$ & $R L$ & $\mathrm{E}$ & India \\
\hline RG117 & IG 65(EC 729024- 120958) & GODA HEENATI::IRGC 31393-1 & SRILANKA & । & indica & $\mathbb{R}$ & $\mathrm{E}$ & Philippines \\
\hline RG118 & Ponmani samba & Landrace & Tamil Nadu, India & $\mathrm{T}$ & indica & $\mathbb{R}$ & M & India \\
\hline RG119 & Ganthasala & Landrace & Tamil Nadu, India & $\mathrm{T}$ & indica & $\mathbb{R}$ & M & India \\
\hline RG120 & Thattan samba & Landrace & Tamil Nadu, India & $\mathrm{T}$ & indica & $\mathbb{R}$ & $\mathrm{E}$ & India \\
\hline RG121 & IG 74(EC 728622- 117517) & $\begin{array}{l}\text { KINANDANG PATONG::IRGC } \\
23364-1\end{array}$ & IRRI, Philippines & । & japonica & $R L$ & M & Philippines \\
\hline RG122 & Kaliyan samba & Landrace & Tamil Nadu, India & $\mathrm{T}$ & indica & $\mathbb{R}$ & M & India \\
\hline RG123 & IG 2(EC 729808-121874) & BLUEBONNET 50:IIRGC 1811-1 & IRRI, Philippines & । & japonica & UP & M & Philippines \\
\hline RG124 & IG 29(EC 728925- 117920) & TOX 782-20-1 & NIGERIA & $\mathrm{T}$ & $\begin{array}{l}\text { Tropical } \\
\text { Japonica }\end{array}$ & $\mathbb{R}$ & $\mathrm{E}$ & Philippines \\
\hline
\end{tabular}


Table 6 Germplasm accessions used in the study (Continued)

\begin{tabular}{|c|c|c|c|c|c|c|c|c|}
\hline RG125 & RPHP 55 & Kalinga -3 & Orissa & 1 & indica & $R L$ & $E$ & India \\
\hline RG126 & Kallimadayan & Landrace & Tamil Nadu, India & $\mathrm{T}$ & indica & $R L$ & E & India \\
\hline RG127 & IG 10(EC 729686- 121743) & HASAN SERAI & IRRI, Philippines & । & aromatic & $\mathbb{R}$ & $\mathrm{E}$ & Philippines \\
\hline RG128 & IG 75(EC 728587- 117420) & AEDAL::IRGC 55441-1 & Korea & $\mathrm{T}$ & japonica & $\mathbb{R}$ & $\mathrm{E}$ & Philippines \\
\hline RG129 & IG 38(EC 728742 - 117707) & DELREX & UNITED STATES & & $\begin{array}{l}\text { Tropical } \\
\text { japonica }\end{array}$ & $\mathbb{I R}$ & M & Philippines \\
\hline RG130 & IG 39(EC 728779- 117750) & HONDURAS & HONDURAS & & indica & $\mathbb{R}$ & M & Philippines \\
\hline RG131 & RPHP 90 & 182(M) & Andhra Pradesh & I & indica & $\mathbb{R}$ & $E$ & India \\
\hline RG132 & IG 33(EC 728938- 117935) & WC 3397 & JAMAICA & & $\begin{array}{l}\text { Tropical } \\
\text { Japonica }\end{array}$ & $\mathbb{R}$ & $\mathrm{E}$ & Philippines \\
\hline RG133 & IG 42(EC 728798- 117774) & KALUBALA VEE & SRILANKA & $\mathrm{T}$ & indica & $\mathbb{I R}$ & $E$ & Philippines \\
\hline RG134 & IG 9(EC 729682- 121739) & $\begin{array}{l}\text { GEMJYA JYANAM::IRGC } \\
\text { 32411-C1 }\end{array}$ & IRRI, Philippines & I & indica & $\mathbb{R}$ & $\mathrm{E}$ & Philippines \\
\hline RG135 & RPHP 161 & Champa Khushi & Vietnam & $\mathrm{T}$ & indica & UP & $E$ & India \\
\hline RG136 & IG 8(EC 729601- 121651) & XI YOU ZHAN:IRGC 78574-1 & China & I & indica & $\mathbb{R}$ & E & Philippines \\
\hline RG137 & IG 37(EC 728715- 117678) & CENIT & ARGENTINA & $\mathrm{T}$ & $\begin{array}{l}\text { Tropical } \\
\text { Japonica }\end{array}$ & $\mathbb{I R}$ & $L$ & Philippines \\
\hline RG138 & Sigappu kuruvikar & Landrace & Tamil Nadu, India & $\mathrm{T}$ & indica & $R L$ & $E$ & India \\
\hline RG139 & RPHP 138 & EDAVANKUDI POKKALI & Kerala, India & $\mathrm{T}$ & indica & Deep water & $L$ & India \\
\hline RG140 & Raja mannar & Landrace & Tamil Nadu, India & $\mathrm{T}$ & indica & $\mathbb{R}$ & M & India \\
\hline RG141 & IG 44(EC 728762- 117729) & EDITH & UNITED STATES & $\mathrm{T}$ & indica & $\mathbb{R}$ & $E$ & Philippines \\
\hline RG142 & Sasyasree & TKM $6 \times 1 R 8$ & West Bengal & I & indica & $\mathbb{R}$ & $\mathrm{E}$ & India \\
\hline RG143 & IG 46(IC 471826- 117647) & BABER & INDIA & I & indica & $\mathbb{R}$ & $E$ & India \\
\hline RG144 & Chetty samba & Landrace & Tamil Nadu, India & $\mathrm{T}$ & indica & $\mathbb{R}$ & E & India \\
\hline RG145 & IG 60(EC 728730- 117695) & CREOLE & Belize & $\mathrm{T}$ & indica & $\mathbb{R}$ & M & Philippines \\
\hline RG146 & IR 75862-206 & IR 75083 X IR 65600 -81-5-3-2 & IRRI, Philippines & I & $\begin{array}{l}\text { Tropical } \\
\text { Japonica }\end{array}$ & $\mathbb{R}$ & M & Philippines \\
\hline RG147 & IG 58(EC 728725- 117689) & Cl 11011 & UNITED STATES & & japonica & $\mathbb{R}$ & M & Philippines \\
\hline RG148 & Chinna aduku nel & Landrace & Tamil Nadu, India & $\mathrm{T}$ & indica & $\mathbb{R}$ & $L$ & India \\
\hline RG149 & RH2-SM-2-23 & SWARNA X MOROBERAKAN & Tamil Nadu, India & 1 & indica & $\mathbb{R}$ & M & India \\
\hline RG150 & IG 14(IC 517381- 121422) & MALACHAN::IRGC 54748-1 & India & I & indica & UP & $E$ & Philippines \\
\hline RG151 & IG 32(EC 728838- 117823) & NOVA & United States & । & japonica & $\mathbb{R}$ & M & Philippines \\
\hline RG152 & RPHP 47 & Pathara (CO-18 x Hema) & India & I & indica & $\mathbb{R}$ & $E$ & India \\
\hline RG153 & Sembilipiriyan & Landrace & Tamil Nadu, India & $\mathrm{T}$ & indica & $R L$ & M & India \\
\hline
\end{tabular}


Table 6 Germplasm accessions used in the study (Continued)

\begin{tabular}{|c|c|c|c|c|c|c|c|c|}
\hline RG154 & IG 48(EC 729203- 121195) & DINOLORES:IIRGC 67431-1 & IRRI, Philippines & I & indica & UP & $M$ & Philippines \\
\hline RG155 & Sona mahsuri & Landrace & Tamil Nadu, India & $\mathrm{T}$ & indica & $\mathbb{R}$ & E & India \\
\hline RG156 & IG 12(EC 729626- 121681) & SHESTAK:IRGC 32351-1 & Iran & । & indica & $\mathbb{R}$ & $\mathrm{E}$ & Philippines \\
\hline RG157 & Karungan & Landrace & Tamil Nadu, India & $\mathrm{T}$ & indica & $\mathbb{R}$ & $\mathrm{E}$ & India \\
\hline RG158 & IG 13(EC 729640- 121696) & CURINCA::C1 & BRAZIL & 1 & indica & IR & $\mathrm{E}$ & Philippines \\
\hline RG159 & Sembala & Landrace & Tamil Nadu, India & $\mathrm{T}$ & indica & $\mathbb{R}$ & $L$ & India \\
\hline RG160 & IG 72(EC 728650- 117587) & TD 25::IRGC 9146-1 & Thailand & । & indica & $\mathbb{R}$ & M & Philippines \\
\hline RG161 & Panamarasamba & Landrace & Tamil Nadu, India & $T$ & indica & $\mathbb{R}$ & M & India \\
\hline RG162 & IR 64 & $\begin{array}{l}\text { IR-5857-33-2-1 x } \\
\text { IR-2061-465-1-5-5 }\end{array}$ & IRRI, Philippines & । & indica & IR & $\mathrm{E}$ & Philippines \\
\hline RG163 & Mikuruvai & Landrace & Tamil Nadu, India & $\mathrm{T}$ & indica & $\mathrm{RL}$ & $\mathrm{E}$ & India \\
\hline RG164 & Thillainayagam & Landrace & Tamil Nadu, India & $\mathrm{T}$ & indica & $\mathbb{R}$ & M & India \\
\hline RG165 & ARB 64 & Variety & Karnataka & I & indica & $\mathbb{R}$ & $\mathrm{E}$ & India \\
\hline RG166 & RPHP 140 & VYTILLA ANAKOPON & Kerala & $\mathrm{T}$ & indica & $\mathbb{R}$ & $\mathrm{E}$ & India \\
\hline RG167 & IG 70(EC 729045- 120983) & IR43:-IRGC 117005-1 & IRRI, Philippines & I & indica & $\mathbb{R}$ & M & Philippines \\
\hline RG168 & Haladichudi & Landrace & Orissa, India & $\mathrm{T}$ & indica & $\mathbb{R}$ & $\mathrm{E}$ & India \\
\hline RG169 & IG 24(EC 728751- 117718) & DNJ 140 & BANGLADESH & । & Aus & $\mathbb{R}$ & $\mathrm{E}$ & Philippines \\
\hline RG170 & RPHP 42 & Salimar Rice -1 & JAMMU \& KASHMIR & । & indica & $\mathbb{R}$ & M & India \\
\hline RG171 & RPHP 44 & BR- 2655 & KARNATAKA & I & indica & $\mathbb{R}$ & $\mathrm{L}$ & India \\
\hline RG172 & IG 25(EC 729728- 121785) & $\begin{array}{l}\text { LOHAMBITRO 224::GERVEX } \\
\text { 5144-C1 }\end{array}$ & Madagascar & । & $\begin{array}{l}\text { Tropical } \\
\text { Japonica }\end{array}$ & $\mathbb{R}$ & $\mathrm{E}$ & Philippines \\
\hline RG173 & IG 73(EC 728627- 117527) & MAKALIOKA 34:IIRGC 6087-1 & IRRI, Philippines & I & indica & $\mathbb{R}$ & E & Philippines \\
\hline RG174 & IG 51(EC 728772- 117742) & GOGO LEMPUK & Indonesia & & $\begin{array}{l}\text { Tropical } \\
\text { Japonica }\end{array}$ & $\mathbb{R}$ & M & Philippines \\
\hline RG175 & Vellai kudaivazhai & Landrace & Tamil Nadu, India & $\mathrm{T}$ & indica & $\mathrm{RL}$ & M & India \\
\hline RG176 & Kodai & Landrace & Tamil Nadu, India & $T$ & indica & $\mathrm{RL}$ & $\mathrm{E}$ & India \\
\hline RG177 & Kallundaikar & Landrace & Tamil Nadu, India & $\mathrm{T}$ & indica & UP & M & India \\
\hline RG178 & IG 17(EC 728900- 117889) & SIGADIS & INDONESIA & $\mathrm{T}$ & indica & $\mathrm{RL}$ & $\mathrm{L}$ & Philippines \\
\hline RG179 & Avasara samba & Landrace & Tamil Nadu, India & $\mathrm{T}$ & indica & $\mathbb{R}$ & $\mathrm{E}$ & India \\
\hline RG180 & IG 59(EC 728729- 117694) & COPPOCINA & BULGARIA & 1 & $\begin{array}{l}\text { Tropical } \\
\text { Japonica }\end{array}$ & $\mathbb{R}$ & M & Philippines \\
\hline RG181 & IG 52(EC 728756- 117723) & DOURADO AGULHA & BRAZIL & । & $\begin{array}{l}\text { Tropical } \\
\text { Japonica }\end{array}$ & $\mathbb{R}$ & M & Philippines \\
\hline RG182 & ARB 59 & Variety & Karnataka & I & indica & $\mathbb{I R}$ & $\mathrm{E}$ & India \\
\hline
\end{tabular}


Table 6 Germplasm accessions used in the study (Continued)

\begin{tabular}{|c|c|c|c|c|c|c|c|c|}
\hline RG183 & RPHP 163 & Seeta sail & West Bengal & 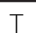 & indica & Scented & M & India \\
\hline RG184 & IG 18(EC 728892- 117880) & SERATOES HARI & INDONESIA & $\mathrm{T}$ & indica & $\mathbb{R}$ & $E$ & Philippines \\
\hline RG185 & RPHP 36 & TKM-9 & Tamil Nadu, India & । & indica & $\mathbb{R}$ & $E$ & India \\
\hline RG186 & IG 28(EC 728920- 117914) & TIA BURA & INDONESIA & $\mathrm{T}$ & $\begin{array}{l}\text { Tropical } \\
\text { Japonica }\end{array}$ & $\mathbb{R}$ & M & Philippines \\
\hline RG187 & Vadakathi samba & Landrace & Tamil Nadu, India & T & indica & $\mathbb{R}$ & M & India \\
\hline RG188 & RPHP 80 & $24(K)$ & Andhra Pradesh & I & indica & $\mathbb{R}$ & $E$ & India \\
\hline RG189 & IG 41(EC 728800- 117776) & KANIRANGA & Indonesia & $\mathrm{T}$ & $\begin{array}{l}\text { Tropical } \\
\text { japonica }\end{array}$ & $\mid \mathrm{R}$ & M & Philippines \\
\hline RG190 & IG 26(IC 0590943- 121899) & BASMATI 370:IRGC 3750-1 & IRRI, Philippines & । & aromatic & $\mathbb{R}$ & $E$ & Philippines \\
\hline RG191 & IG 15(EC 728910- 117901) & SZE GUEN ZIM & CHINA & । & indica & $\mathbb{R}$ & $E$ & Philippines \\
\hline RG192 & Nootri pathu & Landrace & Tamil Nadu, India & T & indica & $R L$ & $\mathrm{~L}$ & India \\
\hline
\end{tabular}

IRRI lines - The number after hyphen inside brackets represent IRGC number 
by the farmers in different parts of the state. Ecological and evolutionary history contributes for the genetic diversity maintained in a population. The varieties with diverse ecosystems and wide eco-geographical conditions contribute for the genetic diversity among rice varieties in this population.

For establishing a core collection for association studies, two step approach followed by Breseghello and Sorrells (2006) and Courtois et al. (2012) was used. This approach involves the determination of population structure and then sampling can be done based on the relatedness of the accessions in the population. Those accessions that show high magnitude of genetic relatedness can be eliminated to develop core collection with diverse representatives. Based on this idea, out of 192 accessions, 150 (Table 5) were selected to form association mapping panel which can be utilized either by genome wide or candidate gene specific association mapping for linking the genotypic and phenotypic variation.

\section{Conclusion}

This study analyze the pattern of divergence exists in a population of 192 rice accessions that constitute our rice diversity panel for association mapping. Based on various statistical methods, we identified two sub groups within 192 rice accessions selected for establishing association mapping panel. The average number of alleles per locus and gene diversity has indicated the existence of broad genetic base in this collection. The result of structure analysis is in accordance with clustering method of neighbor joining tree and principal coordinate analysis. Thus, the results of this study which indicates the genetic diversity of the accessions can be utilized to predict approaches such as association analysis, classical mapping population development; parental line selection in breeding programs and hybrid development for exploiting the natural genetic variation exists in this population.

\section{Methods}

\section{Plant Material}

A collection consisting of 192 rice accessions was used in this study, which consist of land races and varieties collected from nine different states of India as well as from Argentina, Bangladesh, Brazil, Bulgaria, China, Colombia, Indonesia, Philippines, Taiwan, Uruguay, Venezuela and United States (Table 6).

\section{Microsatellite Genotyping}

\section{DNA Isolation and PCR Amplification}

DNA was extracted from leaf tissue by grinding with liquid nitrogen using CTAB method (Saghai-Maroof et al. 1984.). It was diluted to a final concentration of $30 \mathrm{ng}^{-1}$ for enabling polymerase chain reactions. DNA amplification parameters such as specificity, efficiency and fidelity are strongly influenced by the components of the PCR reaction and by thermal cycling conditions (CaetanoAnolles and Brant 1991). Therefore, the careful optimization of reaction components and conditions will ultimately result in more reproducible and efficient amplification. The concentrations of primers, template DNA, Master Mix, and annealing temperature was optimized on eight diverse accessions for 156 SSR markers distributed on the 12 chromosomes by modified Taguchi method (Cobb and Clarkson 1994). Microsatellite primer sequences, annealing temperature and chromosomal locations are obtained from GRAMENE database (http:// archive.gramene.org/markers/microsat/). Sixty one SSR primer pairs which produce polymorphic allele amplification were chosen to genotype the entire set of germplasm collection.

The volume of the PCR reaction system was $10 \mu \mathrm{l}$. The PCR reaction mixture of $10 \mu \mathrm{l}$ had $0.4 \mathrm{mM}$ dNTPs, $4 \mathrm{mM}$ of $\mathrm{MgCl}_{2}, 150 \mathrm{mM}$ of Tris- $\mathrm{HCl}, 10$ pmoles of forward and reverse primer and $0.05 \mathrm{U}$ Taq polymerase with $30 \mathrm{ng}$ of DNA. Polymerase chain reaction was performed in BIORAD THERMAL CYCLER using the following program: $94{ }^{\circ} \mathrm{C}$ for 2 min, 35 cycles of $94{ }^{\circ} \mathrm{C}$ for $45 \mathrm{sec}, 50-60{ }^{\circ} \mathrm{C}$ for $1 \mathrm{~min}, 72{ }^{\circ} \mathrm{C}$ for 2 min with a final extension of $72{ }^{\circ} \mathrm{C}$ for ten min.

\section{Polyacrylamide Gel Electrophoresis}

Amplified products were size separated in native polyacrylamide gel electrophoresis using $6 \%(w / v)$ polyacrylamide gel according to Sambrook et al. (2001) in vertical electrophoresis tank with $1 \mathrm{X}$ TBE at $150 \mathrm{~V}$. The gel size was determined using standard molecular weight size markers after the bands were detected by silver staining.

\section{Allele Scoring}

The bands were visualized in a cluster of two to six in the stained gels for most of the markers. Based on the expected product size given in the GRAMENE website (Additional file 2: Table S1), the size of the most intensely amplified bands around the expected product size for each microsatellite marker was identified using standard molecular weight size markers (20 bp DNA ladder, GeNeI Company). Then the stained gel was dried and documented using light box. Allele score was given based on the presence of a particular size allele in each of the germplasm. The presence was denoted as 1 and absence of an allele as 0 and it was rechecked manually (Additional file 3: Table S2).

\section{Data Analysis}

A 1/0 matrix was constructed based on the presence and absence of alleles for the set of 61 markers. This SSR genotype data was analyzed for genetic diversity and population structure. 


\section{Genetic Diversity}

For a set of accessions, genetic diversity parameters such as number of alleles per locus, allele frequency, heterozygosity and polymorphic information index (PIC) was estimated using the program POWERMARKER Ver3.25 (Liu and Muse 2005). Allele frequency represents the frequency of particular allele for each marker. Heterozygosity is the proportion of heterozygous individuals in the population. Polymorphic information content that represent the amount of polymorphism within a population was estimated based on Botstein et al. (1980).

To assess genetic structure, model based approach and distance based approach were used. Model based approach was utilized with Structure ver 2.3.4 software (Pritchard et al. 2000). The actual number of subpopulation which is denoted by $\mathrm{K}$ was identified by this method. For that, the project was run with the following parameter set: the possibility of admixture and allele frequency correlated. Run length was given as 150,000 burning period length followed by 150,000 Markov Chain Monte Carlo (MCMC) replication. Each $\mathrm{k}$ value was run for 10 times with $\mathrm{k}$ value varying from 1 to 10 . The optimum $\mathrm{k}$ value was determined by plotting the mean estimate of the $\log$ posterior probability of the data ( $\mathrm{L}(\mathrm{K})$ against the given $\mathrm{K}$ value. True number of subpopulation was identified using the maximal value of $\mathrm{L}$ (K). An adhoc quantity $\Delta \mathrm{K}$ proposed by (Evanno et al. 2005) based on second order rate of change of the likelihood function with respect to $\mathrm{K}$ estimated using Structure Harvester (Earl 2012) has also shown a clear peak at the optimal $\mathrm{K}$ value.

Distance based approach which is based on calculating pair wise distance matrix was computed by calculating a dissimilarity matrix using a shared allele index with DARwin software (Perrier and Jacquemoud-Collet 2006). An unweighted neighbor joining tree was constructed using the calculated dissimilarity index. The genetic distance between accessions was estimated using NEI coefficient (Nei 1972) with bootstrap procedure of resampling (1000) across markers and individuals from allele frequencies. To determine the association among the accessions, unweighted pair group method with arithmetic mean (UPGMA) tree was also drawn using Powermarker and viewed in MEGA 6.0 software (Tamura et al. 2013).

The presence of molecular variance within and between hierarchical population structure estimated by Structure was assessed via Analysis of molecular variance (AMOVA) by Arlequin (Excoffier et al. 2005). F statistics which include $F_{I T}$, deviations from HardyWeinberg expectation across the whole population, $\mathrm{F}_{\text {IS }}$ deviation from Hardy- Weinberg expectation within a population and $\mathrm{F}_{\mathrm{ST}}$, correlation of alleles between subpopulation was calculated using AMOVA approach in
Arlequin. AMOVA and Principal Coordinate analysis of the germplasm set was performed based on Nei (Nei 1973) distance matrix using GenAlEx 6.5 (Peakall and Smouse 2012).

\section{Additional files}

Additional file 1: Figure S1. Allelic pattern of different SSR markers used in this study. (JPG $1.03 \mathrm{MB}$ )

Additional file 2: Table S1. Expected product size obtained from Gramene and observed product size for the SSR markers used in this study. (XLS $170 \mathrm{~kb}$ )

Additional file 3: Table S2. Allele matrix of 192 accessions x 61 SSRs. (XLSX $10 \mathrm{~kb})$

\section{Competing interests}

The authors declare that they have no competing interests.

\section{Authors' contributions}

RoS, WN, RS and SKK prepared the samples. WN carried out the genotyping, data analysis and drafted the manuscript. BAP, GP, KG participated in genotyping and data preparation. RoS designed the experiment and revised the manuscript. SD, RS, RM, SM participated in study design and revised the manuscript. All authors read and approve the final manuscript.

\section{Acknowledgement}

This work was supported by a grant from Department of Biotechnology, Government of India under Rice biofortification with enhanced iron and zinc in high yielding non basmati cultivars through marker assisted breeding and transgenic approaches- Phase II (E28SO) scheme. I thank Dr. Yasodha from Institute of Forest Genetics and Tree Breeding, Coimbatore for helping in the analysis.

\section{Author details}

${ }^{1}$ Plant Molecular Biology, Plant Breeding and Genetics Divison, International Rice Research Institute, Manila, Philippines. ${ }^{2}$ Centre for Plant Breeding and Genetics, Tamil Nadu Agricultural University, Coimbatore, India. ${ }^{3}$ Centre for Plant Molecular Biology and Biotechnology, Tamil Nadu Agricultural University, Coimbatore, India. ${ }^{4}$ Crop Physiology laboratory, International Crops Research Institute for the Semi-Arid-Tropics, Hyderabad, India. ${ }^{5}$ International Crops Research Institute for the Semi-Arid-Tropics, Hyderabad, India. ${ }^{6}$ Centre of Excellence in Molecular Breeding, Tamil Nadu Agricultural University, Coimbatore, India.

Received: 30 October 2014 Accepted: 8 August 2015

Published online: 26 September 2015

\section{References}

Abdurakhmonov IY, Abdukarimov A (2008) Application of association mapping to understanding the genetic diversity of plant germplasm resources. Int J Plant Genomics 2008:574927

Agrama H, Eizenga G (2008) Molecular diversity and genome-wide linkage disequilibrium patterns in a worldwide collection of Oryza sativa and its wild relatives. Euphytica 160(3):339-355

Agrama H, Eizenga G, Yan W (2007) Association mapping of yield and its components in rice cultivars. Mol Breed 19(4):341-356

Agrama HA, Yan W, Jia M, Fjellstrom R, McClung AM (2010) Genetic structure associated with diversity and geographic distribution in the USDA rice world collection. Nat Sci 2(04):247

Botstein D, White RL, Skolnick M, Davis RW (1980) Construction of a genetic linkage map in man using restriction fragment length polymorphisms. Am J Hum Genet 32(3):314

Breseghello F, Sorrells ME (2006) Association mapping of kernel size and milling quality in wheat (Triticum aestivum L.) cultivars. Genetics 172(2):1165-1177 Caetano-Anolles G, Brant B (1991) DNA amplification fingerprinting using very short arbitrary oligonucleotide primers. Nat Biotechnol 9(6):553-557 
Caicedo AL, Williamson SH, Hernandez RD, Boyko A, Fledel-Alon A, York TL, Polato NR, Olsen KM, Nielsen R, McCouch SR (2007) Genome-wide patterns of nucleotide polymorphism in domesticated rice. PLoS Genet 3(9):e163

Chakhonkaen S, Pitnjam K, Saisuk W, Ukoskit K, Muangprom A (2012) Genetic structure of Thai rice and rice accessions obtained from the international rice research institute. Rice 5(1):19

Chen H, He H, Zou Y, Chen W, Yu R, Liu X, Yang Y, Gao Y-M, Xu J-L, Fan L-M (2011) Development and application of a set of breeder-friendly SNP markers for genetic analyses and molecular breeding of rice (Oryza sativa L.). Theor Appl Genet 123(6):869-879

Choudhury B, Khan ML, Dayanandan S (2013) Genetic structure and diversity of indigenous rice (Oryza sativa) varieties in the Eastern Himalayan region of Northeast India. Springer Plus 2(1):228

Cobb BD, Clarkson JM (1994) A simple procedure for optimising the polymerase chain reaction (PCR) using modified Taguchi methods. Nucleic Acids Res 22(18):3801-3805

Courtois B, Frouin J, Greco R, Bruschi G, Droc G, Hamelin C, Ruiz M, Clément G, Evrard J-C, van Coppenole S (2012) Genetic diversity and population structure in a European collection of rice. Crop Sci 52(4):1663-1675

Das B, Sengupta S, Parida SK, Roy B, Ghosh M, Prasad M, Ghose TK (2013) Genetic diversity and population structure of rice landraces from Eastern and North Eastern States of India. BMC Genet 14(1):71

Deepa G, Singh V, Naidu KA (2008) Nutrient composition and physicochemical properties of Indian medicinal rice-Njavara. Food Chem 106(1):165-171

Earl DA (2012) STRUCTURE HARVESTER: a website and program for visualizing STRUCTURE output and implementing the evanno method. Conserv Genet Resour 4(2):359-361

Ebana K, Kojima Y, Fukuoka S, Nagamine T, Kawase M (2008) Development of mini core collection of Japanese rice landrace. Breed Sci 58(3):281-291

Evanno G, Regnaut S, Goudet J (2005) Detecting the number of clusters of individuals using the software STRUCTURE: a simulation study. Mol Ecol 14(8):2611-2620

Excoffier L, Laval G, Schneider S (2005) Arlequin (version 3.0): an integrated software package for population genetics data analysis. Evol Bioinformatics Online 1:47

Gao L-Z, Zhang C-H, Chang L-P, Jia J-Z, Qiu Z-E, Dong Y-S (2005) Microsatellite diversity within Oryza sativa with emphasis on indica-japonica divergence. Genet Res 85(01):1-14

Garris AJ, Tai TH, Coburn J, Kresovich S, McCOUCH S (2005) Genetic structure and diversity in Oryza sativa L. Genetics 169(3):1631-1638

Hesham AA, Yan W, Fjellstrom R, Jia M, McClung A (2008) Genetic diversity and relationships assessed by SSRs in the USDA World-Wide Rice Germplasm Collection. In: The 2008 joint annual meeting

Horst L, Wenzel G (2007) Molecular marker systems in plant breeding and crop improvement, vol 55. Springer, Berlin

Jin L, Lu Y, Xiao P, Sun M, Corke H, Bao J (2010) Genetic diversity and population structure of a diverse set of rice germplasm for association mapping. Theor Appl Genet 121(3):475-487

Krishnanunni K, Senthilvel P, Ramaiah S, Anbarasu A (2015) Study of chemical composition and volatile compounds along with in-vitro assay of antioxidant activity of two medicinal rice varieties: Karungkuravai and Mappilai samba. Journal of Food Science and Technology 52 (5):2572-2584. doi:10.1007/ s13197-014-1292-z

Lapitan VC, Brar DS, Abe T, Redoña ED (2007) Assessment of genetic diversity of Philippine rice cultivars carrying good quality traits using SSR markers. Breed Sci 57(4):263-270

Liakat Ali M, McClung AM, Jia MH, Kimball JA, McCouch SR, Georgia CE (2011) A rice diversity panel evaluated for genetic and agro-morphological diversity between subpopulations and its geographic distribution. Crop Sci 51(5):2021-2035

Liu K, Muse SV (2005) PowerMarker: an integrated analysis environment for genetic marker analysis. Bioinformatics 21(9):2128-2129

Lu H, Redus MA, Coburn JR, Rutger JN, McCOUCH SR, Tai TH (2005) Population structure and breeding patterns of 145 US rice cultivars based on SSR marker analysis. Crop Sci 45(1):66-76

McCouch SR, Chen X, Panaud O, Temnykh S, Xu Y, Cho YG, Huang N, Ishii T, Blair M (1997) Microsatellite marker development, mapping and applications in rice genetics and breeding. Plant Mol Biol 35(1-2):89-99
Nachimuthu W, Robin S, Sudhakar D, Rajeswari S, Raveendran M, Subramanian K, Tannidi S, Pandian BA (2014) Genotypic variation for micronutrient content in traditional and improved rice lines and its role in biofortification programme. Indian J Sci Technol 7(9):1414-1425

Nei M (1972) Genetic distance between populations. Am Nat 106:283-292

Nei M (1973) Analysis of gene diversity in subdivided populations. Proc Natl Acad Sci 70(12):3321-3323

Ni J, Colowit PM, Mackill DJ (2002) Evaluation of genetic diversity in rice subspecies using microsatellite markers. Crop Sci 42(2):601-607

Peakall R, Smouse PE (2012) GenAlEx 6.5: genetic analysis in excel. Population genetic software for teaching and research-an update. Bioinformatics 28(19):2537-2539

Perrier X, Jacquemoud-Collet J (2006) DARwin software

Powell W, Morgante M, Andre C, Hanafey M, Vogel J, Tingey S, Rafalski A (1996) The comparison of RFLP, RAPD, AFLP and SSR (microsatellite) markers for germplasm analysis. Mol Breed 2(3):225-238

Pritchard JK, Stephens M, Donnelly P (2000) Inference of population structure using multilocus genotype data. Genetics 155(2):945-959

Qi Y, Zhang D, Zhang H, Wang M, Sun J, Wei X, Qiu Z, Tang S, Cao Y, Wang X (2006) Genetic diversity of rice cultivars (Oryza sativa L.) in China and the temporal trends in recent fifty years. Chinese Sci Bull 51(6):681-688

Qi Y, Zhang H, Zhang D, Wang M, Sun J, Ding L, Wang F, Li Z (2009) Assessing indica japonica differentiation of improved rice varieties using microsatellite markers. J Genet Genomics 36(5):305-312

Ram SG, Thiruvengadam V, Vinod KK (2007) Genetic diversity among cultivars, landraces and wild relatives of rice as revealed by microsatellite markers. J Appl Genet 48(4):337-345

Saghai-Maroof MA, Soliman KM, Jorgensen AR, Allard RW (1984) Ribosomal DNA spacer length polymorphisms in barley: Mendelian inheritance, chromosomal location and population dynamics. Proc Natl Acad Sci U S A 81:8014-8018

Sambrook J, Russell DW, Russell DW (2001) Molecular cloning: a laboratory manual (3-volume set), vol 999. Cold spring harbor laboratory press, Cold Spring Harbor, New York

Sow M, Ndjiondjop M-N, Sido A, Mariac C, Laing M, Bezançon G (2014) Genetic diversity, population structure and differentiation of rice species from Niger and their potential for rice genetic resources conservation and enhancement. Genet Resour Crop Evol 61(1):199-213

Tamura K, Stecher G, Peterson D, Filipski A, Kumar S (2013) MEGA6: molecular evolutionary genetics analysis version 6.0. Mol Biol Evol 30(12):2725-2729

Thornsberry JM, Goodman MM, Doebley J, Kresovich S, Nielsen D, Buckler ES (2001) Dwarf8 polymorphisms associate with variation in flowering time. Nat Genet 28(3):286-289

Valarmathi R, Raveendran M, Robin S, Senthil N (2015) Unraveling the nutritional and therapeutic properties of 'Kavuni' a traditional rice variety of Tamil Nadu. Journal of Plant Biochemistry and Biotechnology 24 (3):305-315. doi:10.1007/ s13562-014-0274-6

Varshney RK, Chabane K, Hendre PS, Aggarwal RK, Graner A (2007) Comparative assessment of EST-SSR, EST-SNP and AFLP markers for evaluation of genetic diversity and conservation of genetic resources using wild, cultivated and elite barleys. Plant Sci 173(6):638-649

Zhang D, Zhang H, Wei X, Qi Y, Wang M, Sun J, Ding L, Tang S, Cao Y, Wang X (2007) Genetic structure and diversity of Oryza sativa L. in Guizhou, China. Chinese Sci Bull 52(3):343-351

Zhang D, Zhang H, Wang M, Sun J, Qi Y, Wang F, Wei X, Han L, Wang X, Li Z (2009) Genetic structure and differentiation of Oryza sativa L. in China revealed by microsatellites. Theor Appl Genet 119(6):1105-1117

Zhang P, Li J, Li X, Liu X, Zhao X, Lu Y (2011) Population structure and genetic diversity in a rice core collection (Oryza sativa L.) investigated with SSR markers. PLoS One 6(12):e27565

Zhao K, Tung C-W, Eizenga GC, Wright MH, Ali ML, Price AH, Norton GJ, Islam MR, Reynolds A, Mezey J (2011) Genome-wide association mapping reveals a rich genetic architecture of complex traits in Oryza sativa. Nat Commun 2:467

Zhao K, Wright M, Kimball J, Eizenga G, McClung A, Kovach M, Tyagi W, Ali ML, Tung C-W, Reynolds A (2010) Genomic diversity and introgression in O. sativa reveal the impact of domestication and breeding on the rice genome. PloS one 5(5):e10780 